\title{
Tauopathy-associated tau modifications selectively impact neurodegeneration and mitophagy in a novel C. elegans single-copy transgenic model
}

\author{
Sanjib Guha', Sarah Fischer', Gail V. W. Johnson ${ }^{1 *}$ and Keith Nehrke ${ }^{2^{*}}$ (1)
}

\begin{abstract}
Background: A defining pathological hallmark of the progressive neurodegenerative disorder Alzheimer's disease (AD) is the accumulation of misfolded tau with abnormal post-translational modifications (PTMs). These include phosphorylation at Threonine 231 (T231) and acetylation at Lysine 274 (K274) and at Lysine 281 (K281). Although tau is recognized to play a central role in pathogenesis of $A D$, the precise mechanisms by which these abnormal PTMs contribute to the neural toxicity of tau is unclear.
\end{abstract}

Methods: Human ON4R tau (wild type) was expressed in touch receptor neurons of the genetic model organism C. elegans through single-copy gene insertion. Defined mutations were then introduced into the single-copy tau transgene through CRISPR-Cas9 genome editing. These mutations included T231E, to mimic phosphorylation of a commonly observed pathological epitope, and K274/281Q, to mimic disease-associated lysine acetylation collectively referred as "PTM-mimetics" - as well as a T231A phosphoablation mutant. Stereotypical touch response assays were used to assess behavioral defects in the transgenic strains as a function of age. Genetically-encoded fluorescent biosensors were expressed in touch neurons and used to measure neuronal morphology, mitochondrial morphology, mitophagy, and macro autophagy.

Results: Unlike existing tau overexpression models, C. elegans single-copy expression of tau did not elicit overt pathological phenotypes at baseline. However, strains expressing disease associated PTM-mimetics (T231E and K274/ 281Q) exhibited reduced touch sensation and neuronal morphological abnormalities that increased with age. In addition, the PTM-mimetic mutants lacked the ability to engage neuronal mitophagy in response to mitochondrial stress.

Conclusions: Limiting the expression of tau results in a genetic model where modifications that mimic pathologic tauopathy-associated PTMs contribute to cryptic, stress-inducible phenotypes that evolve with age. These findings and their relationship to mitochondrial stress provides a new perspective into the pathogenic mechanisms underlying AD.

Keywords: Alzheimer's disease, C. elegans, Tau, Neurodegeneration, Post-translational modifications

\footnotetext{
*Correspondence: Gail_Johnsonvoll@urmc.rochester.edu;

Keith_Nehrke@urmc.rochester.edu

'Department of Anesthesiology \& Perioperative Medicine, Box 604, University

of Rochester Medical Center, 601 Elmwood Avenue, Rochester, NY 14642,

USA

2Department of Medicine, Box 675, University of Rochester Medical Center,

Nephrology Division, 601 Elmwood Avenue, Rochester, NY 14642, USA
}

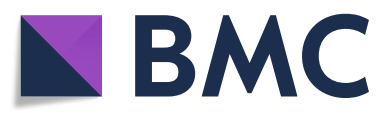

( The Author(s). 2020 Open Access This article is licensed under a Creative Commons Attribution 4.0 International License, which permits use, sharing, adaptation, distribution and reproduction in any medium or format, as long as you give appropriate credit to the original author(s) and the source, provide a link to the Creative Commons licence, and indicate if changes were made. The images or other third party material in this article are included in the article's Creative Commons licence, unless indicated otherwise in a credit line to the material. If material is not included in the article's Creative Commons licence and your intended use is not permitted by statutory regulation or exceeds the permitted use, you will need to obtain permission directly from the copyright holder. To view a copy of this licence, visit http://creativecommons.org/licenses/by/4.0/. The Creative Commons Public Domain Dedication waiver (http://creativecommons.org/publicdomain/zero/1.0/) applies to the data made available in this article, unless otherwise stated in a credit line to the data. 


\section{Background}

Alzheimer's disease (AD) is the most common degenerative brain disease in the aged population. It is characterized by the progressive decline of cognition and memory, as well as changes in behavior and personality [1]. One of the key pathological hallmarks of $\mathrm{AD}$ is neurofibrillary tangles (NFTs), which are primarily composed of abnormally modified tau [2]. Tau isolated from AD brain exhibits a number of post-translational modifications (PTMs); including increases in phosphorylation and acetylation at specific residues [3, 4]. While it is clear that tau is central to AD pathogenesis, the concept of large insoluble NFTs in AD and in family of related neurodegenerative diseases, called tauopathies, being the principle mediators of neuronal toxicity has been gradually abandoned [5, 6]. Instead, toxicity appears to result from soluble or oligomeric forms of tau that exhibit increased, disease-associated phosphorylation and acetylation at specific residues altering its turnover and function $[7,8]$.

Studies to date have provided evidence that phosphorylation of tau at Threonine 231 (T231) occurs early in the evolution of tau pathology; for example, increased staining for this epitope is observed in "pre-tangle" neurons [9]. Further, increased phospho-T231 tau was observed in neurons differentiated from iPSCs of sporadic AD cases [10]. Phosphorylation of tau at specific sites causes significant changes in tau structure $[11,12]$ and impairs microtubule binding [13, 14]. In addition, phosphorylation of tau at T231 precedes the formation of tau oligomers $[8,15]$, which likely contribute to tau toxicity [16].

As with phosphorylation, abnormal tau acetylation also likely plays a critical role in tauopathies [17-19]. There are data indicating that acetylation inhibits binding of tau to microtubules, enhances tau accumulation by preventing degradation and promotes the aggregation of tau in neurons [20-22]. In particular, increased expression of tau acetylated at K274 and K281 appears to result in mislocalization of tau, destabilization of the cytoskeleton in the axon initial segment, and synaptic dysfunction [21, 23]. Altogether, these experiments suggest a potential role for tau acetylated at K274/281 in $\mathrm{AD}$ pathogenesis. While these studies indicate that modifications of human tau at specific residues play a pivotal role in mediating tau dysfunction, the precise mechanism by which specific tau PTMs contribute to the toxicity of soluble tau forms is still unclear.

Mitochondrial dysfunction is a characteristic of many neurodegenerative diseases including $\mathrm{AD}[24,25]$, and over expression of human full length tau or mutant human tau contributes to mitochondrial dysfunction in AD animal models [26, 27]. Mitochondria play a complex role in the cell - they not only generate most of the energy needed to support the various neuronal functions [28], but also are mediators of homeostatic processes that are necessary for neuronal health [29]. Although it is likely that tau pathology affects mitochondrial biology, the underlying mechanisms are not well understood, nor it is known how tau modified at disease relevant sites differ from its wild type form in causing mitochondrial abnormalities leading to neurodegeneration.

To understand the role of tau in the context of AD per se, tau transgenic models have been developed in $C$. elegans [30-33], D. melanogaster [34, 35] and mice [36, 37] by overexpression of human wild-type full-length tau [26], tau with mutations that result in frontotemporal lobar degeneration (FTLD) [37], or a tau with a risk factor mutation for AD FTLD such as A152T [32]. Studies utilizing these transgenic animals have made important contributions to the field, but over-expression of tau can potentially lead to synthetic toxic or gain-of-function phenotypes, such as uncoordinated locomotion phenotype, reduced motility in liquid and decreased lifespan [33, 38]. Moreover, tau overexpression strain displays severe mitochondrial dysfunction and decreased mitophagy in young animals [39]. Thus, all these caveats must always be kept in mind when extrapolating results to the human disease $[38,40]$.

Here, we attempted to circumvent the limitations associated with tau overexpression by taking advantage of single-copy genome insertion methodology in the genetic model organism C. elegans [41]. Using this methodology, human tau was expressed in a defined set of mechanosensory neurons that mediate a stereotypical behavioral output [42]. To interrogate the effects of pathologic PTMs in this system, CRIS PR-Cas9 gene editing was used to introduce diseaseassociated phosphorylation mimicking $(\mathrm{T} \rightarrow \mathrm{E})$ or a non-phosphorylatable $(\mathrm{T} \rightarrow \mathrm{A})$ mutation at the $\mathrm{T} 231$ position of the wild-type tau isoform, or alternatively acetylation mimicking $(\mathrm{K} \rightarrow \mathrm{Q})$ mutations at the $\mathrm{K} 274$ and K281 positions of the wild type tau isoform. A combination of behavioral assays and fluorescent biosensors were used to study the impact of tau and mutant tau expression on neuronal morphology and mitochondrial phenotypes, with the advantage of being able to assess age-dependence in a relatively short time frame $[43,44]$.

Our results clearly demonstrate that wild-type tau has little effect at baseline, but that mutations that mimic disease-relevant tau PTMs selectively impact sensory neuron function and morphology and mitochondrial handling. Moreover, age exacerbates defects in one of the tau mutant strains, but not the others. This leads us to conclude that using our single-copy tau model confers the ability to discern between the pathological consequences of individual tau mutants 
with unprecedented precision. Surprisingly, tau mutations that mimic disease-associated PTMs also completely suppressed paraquat-induced mitophagy, supporting the idea that pathological modifications of tau result in dysfunctional responses to stress, including perhaps the stress of aging.

\section{Methods}

\section{Plasmid construction}

Briefly, pBJ1 codes for the fluorescent photo-convertible protein Dendra2 [45], cloned downstream of the mec-7 promoter in a pFH6.II C. elegans expression vector [46]. pBJ2 adds the coding sequence for tau (0N4R) inserted downstream and in-frame with Dendra2. pBJ5 and pBJ6 are derivatives of $\mathrm{pBJ} 1$ and $\mathrm{pBJ} 2$, respectively, with the tau expression cassette sub-cloned into pCFJ151 (Addgene) to generate MosSCI inserts at the ttTi5605 loci in C. elegans chromosome II [47]. pSKG1 contains a mec-4 promoter driving the expression of C. elegans codon-optimized mito-mKeima (courtesy of Dr. C. Rongo, Rutgers University). pMH878 (courtesy of Dr. M. Hansen, Sanford Burnham Prebys Medical Discovery Institute) contains a mec-7 promoter driving the expression of C. elegans LGG-1.

\section{C. elegans strain generation}

The wild-type background strain was Bristol-N2. KP4 glr-1 (n2461) and TU253 mec-4 (u253) strains were provided by the Caenorhabditis Genetics Center (CGC). Other strains used here include, KWN177 rnyIs14 [Pmec-4::mCherry], KWN796 rnyEx336 [pSKG1 (Pmec-4::mito-mKeima), $p$ CFJ90 (Pmyo-2::wCherry), $p C I$ (pha-1+)] and KWN 819 rnyEx337 [pSKG7 (Pmec-4:: LGG-1::GFP::mCherry, $\quad$ CFJ90(Pmyo-2:: wCherry),pCI(pha-1+)]. Transgenic strain for tau overexpression in touch neuron include KWN91 rnyEx048 [p BJ5 (Pmec7::V5-tau; unc-119+); pCFJ70; pCFJ90; pJL44] and KWN93 rnyEx086 [p BJ6 (Pmec-7::Dendra2-tau; unc119+); $p$ CFJ70; $p$ CFJ90; $p J L 44]$. Transgenic strains for single-copy gene expression were generated using MosSCI insertion [47] into ttTi5605 on Chromosome II via established protocols [41], and include the following: KWN169, rnySi26 [Pmec-7::Dendra2; unc-119+] II; KWN167, rnySi24 [Pmec-7::Dendra2::Tau-T4; unc-119+ ] II. Both strains were sequenced completely through the insertion site and were outcrossed at least four times to the lab N2-Bristol stock. CRISPR-Cas9 gene editing was used to introduce site-specific mutations into the rnySi24 tau coding region via a co-CRISPR strategy and oligonucleotide-mediated HDR using purified Cas9 RNP injection [48, 49]. Targeting crRNAs were from Dharmacon and were complexed to scaffolding RNAs for Cas9, with genomic recognition sites as follows:

Tau T231, 5'ACGGCGACTTGGGTGGAGTA3'; Tau K274/281, 5'GCACCAGCCGGGAGGCGGGA3'.

Single stranded oligonucleotide directed repair templates were:

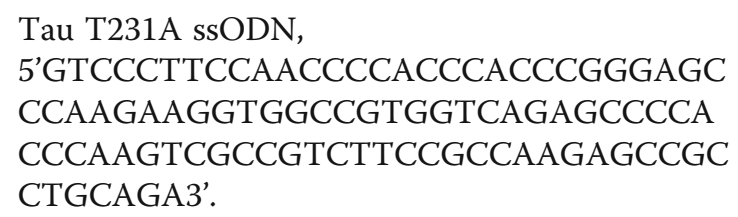

Tau T231E ssODN, 5'GTCCCTTCCAACCCCACCCACCCGGGAGCC CAAGAAGGTGGCCGTGGTCAGAGAGCCA CCCAAGTCGCCGTCTTCCGCCA AGAGCCGCCTGCAGA3'.

Tau K274/281Q ssODN, 5'CGGCTCCACTGAGAACCTGAAGCACCAGCC GGGAGGCGGGCAAGTGCAGATAATTAATAAG CAGCTGGATCTTAGCAACGTCCAGTCC AAGTGTGGCTCAAAGGATA3’.

In all cases, HDR would be predicted to disrupt the PAM, but leave the coding sequence potential outside of the desired amino acid substitution intact. Repair at T231 also disrupted a BtsaI site, while repair at K274/281 created a new PvulI site. These modifications could be detected via restriction analysis of genomic PCR products and were used to screen $d p y-10$ co-CRISPR mutants for edits with primers:

\section{Tau geno-F1, 5' -AAAGACACCACCCAGCTCTG-3'.}

\section{Tau geno-R1, 5'TGTTGCCTAATGAGCCACAC3',}

Following isolation of homozygous tau mutants, editing was confirmed via genomic PCR sequencing, and the mutants were crossed out of the co-CRISPR'd $d p y-10$ mutant background. The final strains are KWN788 rnySi51 [Tau-T4 (T231A) *rnySi24] II, KWN789 rnySi52 [Tau-T4 (T231E) "rnySi24] II, KWN790 rnySi53 [Tau-T4 (K274Q; K281Q) "rnySi24] II. For crossing tau MosSCI strains into various genetic backgrounds, Dendra2 fluorescent was used to guide selection of homozygous mutants, and PCR genotyping was used to confirm homozygosity with primers specific to the ttTi5605 loci, including:

MosSCI ttTi5605-F, 5’GTTTTTGATTGCGTG CGTTA3'.

MosSCI ttTi5605-R, 5’ACATGCTTCGTGCAAAACAG3'. 
MosSCI ttTi5605 insert-F, 5'CATCCCGGTTTCTGT CAAAT3'.

Other strains included KWN791 rnySi51 II; rnyIs14, KWN797 rnySi26 II; rnyIs14 KWN798 rnySi24 II, rnyIs14, KWN800 rnySi52 II; rnyIs14, KWN801 rnySi53 II; rnyIs14 KWN802 rnySi26 II; rnyEx336 KWN803 rnySi24 II; rnyEx336, KWN804 rnySi52 II; rnyEx336, KWN805 rnySi53 II; rnyEx336, KWN806 rnySi51 II; rnyEx336.

\section{C. elegans strains growth and maintenance}

Nematodes were maintained at $20^{\circ} \mathrm{C}$ on Nematode Growth Media (NGM) plates made with Bacto Agar (BD Biosciences). The plates were seeded with live E. coli OP50-1 bacterial strain (cultured overnight at $37^{\circ} \mathrm{C}$ at $220 \mathrm{rpm}$ ) and allowed to grow overnight, as previously described [50]. For experimental assays, after synchronization by standard procedure with sodium hypochlorite, 4th larval stage (L4) hermaphrodites (characterized by the appearance of a "Christmas tree vulva") were selected and moved to test plates. The day after moving was considered adult day 1 , and animals were assayed on day 3 and day 10 . Animals were transferred daily to avoid mixed population until they stop laying eggs.

\section{Blinding of experiments and replicates}

Insofar as possible, experimentalists were blinded to genotype. Data in the figures generally represents the pooled results of three experimental replicates with either two technical replicates per condition or two independent researchers blindly analyzing the data, with the total number of animals or neurons scored reported as $\mathrm{N}$, as indicated.

\section{RNA isolation and quantitative real-time polymerase chain reaction (qRT-PCR) of tau (ON4R)}

RNA was isolated from 400 to 500 adult N2, Dendra2 and tau transgenic worms grown at $20^{\circ} \mathrm{C}$. The worms were washed three times with $1 \mathrm{X}$ M9 buffer to remove any attached bacteria and finally collected in $300 \mathrm{ul}$ RNAase free water. Before RNA isolation, worms were freeze/thawed using dry ice and ethanol and subsequently processed using QIAShredder, Qiagen. RNA was isolated using Qiagen RNeasy plus mini kit. The RNA concentration was quantified using a NanoDrop 2000 Spectrophotometer. A total of $200 \mathrm{ng}$ RNA was reversetranscribed to cDNA using the Verso cDNA Synthesis Kit, Thermo Scientific. Real-time PCR was then performed using SYBR green master mix in a BIO-RAD MyCycler system. Relative gene expression was calculated by the $2^{-\Delta \Delta \mathrm{Ct}}$ method. The experiment was performed in three biological replicates, with four technical replicates of each sample and using two housekeeping genes (act-1 and pmp-2). The final data was averaged and represented in comparison to wild type tau (0N4R). The sequence of the qRT primers used for the analysis are as follows:

\begin{tabular}{ll}
\hline act-1 RTF & $5^{\prime}$ - CAA CAC TGT TCT TTC CGG AG - 3' \\
act-1 RTR & 5' - CCT GAT CTT CAT GGT TGA TGG G - 3' \\
pmp-2 Forward-Q & 5' - ATC TTT CAA AGC CAA TCC TCG AC - 3' \\
pmp-2 Reverse-Q & $5^{\prime}$ - GAG ATA AGT CAG CCC AAC TCC - 3' \\
ON4R-For-Q-106 bp & $5^{\prime}$ - CCA AGT GTG GCT CAT TAG GCA - 3' \\
ON4R-Rev-Q-106 bp & $5^{\prime}$ - CCA ATC TTC GAC TGG ACT CTG T - 3' \\
ON4R-For-Q-128 bp & $5^{\prime}$ - GGG GGC TGATGG TAA AAC GA - 3' \\
ON4R-Rev-Q-128 bp & $5^{\prime}$ - CAG AGC TGG GTG GTG TCT TT - 3' \\
\hline
\end{tabular}

\section{Locomotory rate assay}

Assay plates were prepared using standard procedures [51]. Synchronized day 3 and day 10 adult animals were assayed for the actual experiment. For well-fed animals, locomotory rate was measured by removing 5 animals from original plate and transferring them to an assay plate. Five minutes after transfer, the number of body bends in $20 \mathrm{~s}$ intervals was sequentially counted for each of the 5 animals on the assay plate and this was repeated for next set of animals using a different assay plate.

\section{Thrashing assay}

A drop of $2 \%$ agarose (ultraPURE ${ }^{\oplus}$ agarose) was poured over the glass slide and allowed to dry and then $20 \mu \mathrm{l}$ of M9 was poured on it. Age-synchronized animals were picked to that drop of M9 buffer. After 2 min in M9, thrashing rates were assessed via videography on a stereo dissecting scope. A single thrash was defined as a complete change in the direction of the body down the midline. Animals that were motionless for $10 \mathrm{~s}$ were discarded from the analysis [52].

\section{Touch sensitivity assay}

The behavioral response to being touched by an eyelash was adapted from an assay previously described [53, 54]. The animals were touched anteriorly specifically behind the terminal bulb of the pharynx with the eyelash, 10 times per animal, with a $10 \mathrm{~s}$ gap between each touch. Typically, if the animal demonstrates an omega turn or if it reversed its direction after an anterior touch, the animal was scored as giving positive response. Touch response percentage was generated by the amount of times an animal responded to the touch stimulus over the total number of times they were touched.

\section{Life span analysis}

After alkaline hypochlorite treatment, synchronized L1 animals were placed on freshly grown OP50-1 seeded NGM plates. Fifteen animals from the 4th larval stage 
(L4) were transferred to a small $(35 \mathrm{~mm})$ individual seeded NGM plate with a total 3 plates for each genotype. Each day they were transferred to new plates to avoid mixing of populations until they stopped producing offspring. Simultaneously the worms were counted alive visually or with gentle prodding on the head. Animals were censored in the event of internal hatching of larva, body rupture or crawling off the plate. The experiment was conducted at $20^{\circ} \mathrm{C}$ temperature and scored until all the worms died [55].

\section{Mitochondrial stress assay}

For paraquat (PQT) mild stress assays, synchronized 2day old adult and 9-day old adult hermaphrodites were exposed to $8 \mathrm{mM}$ PQT $[56,57]$ in NGM plate for overnight at $20^{\circ} \mathrm{C}$. Animals were picked from the respective (treated and control) plates the next day and imaged, as described below.

\section{Neurodegeneration assay}

For imaging, animals were mounted by placing them in $3 \%$ agarose pads on glass slides and immobilized with 1 $\mathrm{mM}$ tetramisole hydrochloride (Sigma). Imaging was performed using Confocal Laser Scanning Confocal microscope (Olympus 1X61) and FV10-ASW 4.1 software. All images were acquired under the same exposure conditions with a $20 x$ objective, and for each experimental replicate, all genotypes were represented and imaged that day. In analysis of touch neurons, Pmec-4:: mCherry expressing animals scored positive for the presence of extra neuronal processes when a visible mCherry-labeled branch was observed emanating from the posterior portion of ALM cell body. Similarly, ALM / PLM neuron pairs were scored as overextended when the PLM neurite extended anterior to the ALM cell body [58, 59]. Other defects in axonal morphology were assigned to one of the following classes of neuronal abnormality: broken or gap in the axon structure, blebbed or bead like structure on the axon body, misguided or wavy shaped axon [60].

\section{Mitophagy assay}

A strain containing mito-mKeima [61, 62] expressed specifically in touch cells was used for assay. Animals were mounted on $2 \%$ agarose pads on glass slides and immobilized with $1 \mathrm{mM}$ tetramisole hydrochloride before imaging. Imaging was performed using a Nikon Eclipse inverted microscope coupled to a six channel LED light source (Intelligent Imaging Innovation, Denver, CO), an ORCA-Flash4.0 V2 Digital CMOS camera (Hamamatsu Photonics, Bridgewater Township, NJ) and Slidebook6 software (Intelligent Imaging Innovation, Denver, CO). All images were acquired under the same exposure conditions and each experiment was imaged in one session. The PLM cell body was identified by their position toward the posterior of the animal, near the tail and was focused with a 100x oil immersion lens under visible light using DIC contrast. 600-nm + emissions were captured first following excitation at $550-\mathrm{nm}$ and then immediately thereafter at 440-nm, keeping light intensity and exposure times constant between images. Images were quantified using Slidebook6 software by selecting the ROI, measuring the mean intensities for both channels and subtracting the background intensity. Mitophagy index was obtained by calculating the dual excitation $550-\mathrm{nm} / 440-\mathrm{nm}$ ratio.

\section{Autophagy assay}

Animals were immobilized using the same strategy as above and imaged using a Nikon A1R HD scanning confocal microscope, Z-stack images were acquired at $0.6 \mu \mathrm{m}$ slice intervals at $63 \mathrm{x}$ with NIS-Elements version 5.11 software. GFP excitation/ emission was set to (488/ $550) \mathrm{nm}$ and the mCherry was set to $(561 / 600) \mathrm{nm}$. The intensity of red fluorescence compared to green is stronger and in order to see the mCherry-positive punctae clearly, the gain of the red channel $(H V=5)$ was purposely set lower than that of the green channel $(\mathrm{HV}=10)$, keeping the laser power the same for both. For experiments, punctae was counted in the neurons after Z-stacking all the images using ImageJ software. The average and SD were calculated and data were analyzed using one-way analysis of variance (ANOVA) or two-way ANOVA as applicable and graphs were plotted using GraphPad Prism.

\section{Mitochondrial morphology assay}

Mitochondrial morphology was analyzed using images acquired on a florescence microscopy rig as described above, from animals expressing mito-mKeima in the mechanosensory touch cells, acquired at 440-nm excitation, $600 \mathrm{~nm}+$ emission. The morphological features were categorized into four distinct groups: 1) a network of long interconnected mitochondria with tubular-reticular or normal morphology, 2) visible fragmentation of the network, but lacking aggregates, 3) fragmentation consisting of short round mitochondria, but no more than one visible aggregate, 4) short round mitochondria comprising the majority of the population, with more than one large aggregate [63]. Two investigators independently analyzed subsets of images and compared results to ensure the reproducibility of the analysis.

\section{Statistical analysis}

All statistical analyses were conducted using Prism 8.0 (GraphPad Software), with alpha-error level of $p<0.05$ considered to be significant. Data were averaged and represented as mean \pm standard error (mean \pm SEM) or as mean \pm standard deviation (mean $\pm \mathrm{SD}$ ), depending 
upon the number of experimental replicates. In general, group differences were analyzed with either one-way or two-way ANOVA depending upon the variables. Fisher's exact test was used to obtain $p$-values for the categorical data on pathologic neuronal morphology. Differences in lifespan were assessed by Mantel-Cox log rank analysis, and mitochondrial morphology data, which was categorical with four levels, was assessed using a Wilcoxen signed-rank test. The sample sizes were based on those found previously in the laboratory to provide appropriate power for discerning phenotypic differences among genotypes. Graphs were plotted in Prism 8.0 (GraphPad Software) and Microsoft Excel.

\section{Results}

\section{Single-copy tau mutants that mimic Tauopathy-} associated PTMs impact behavior

Tau expression via conventional extrachromosomal transgenic arrays in C. elegans has been shown to severely impact neuronal morphology and function [31, 40]. Here, in an attempt to circumvent potential caveats related to overexpression, novel transgenic AD models were engineered using single-copy Mos-transposon mediated insertion of a tau expression cassette into the worm genome [41, 47]. The mec-7 promoter was used to drive the expression of $0 \mathrm{~N} 4 \mathrm{R}$ tau $[64,65]$ as a translational fusion with the fluorescent protein Dendra2 [45] in mechanosensory touch neurons ALML/ALMR, AVM, PLML/PLMR, and PVM (Fig. 1e-h), which mediate the behavioral response to light touch. The 0N4R fusion to Dendra2 will be referred to hereafter as TauT4. Dendra2 was also expressed alone (Fig. 1a-d), and this negative control strain responded to light touch similarly to the wild-type $\mathrm{N} 2$ strain at both day 3 and day 10 of adulthood (Fig.
S1A). Surprisingly, in contrast to previously published work where tau was expressed in touch neurons [38], single-copy TauT4 worms exhibited normal touch responsiveness as both young day 3 post-reproductive adults (Fig. 2c) and older day 10 adults (Fig. 2d). However, when an identical TauT4 transgene was expressed from an extrachromosomal multi-copy array, the strain exhibited significant touch sensation defects both at Day 3 and Day 10 (Fig. S1B). Moreover, this strain was $\sim 10$-fold more fluorescent compared to single-copy TauT4 (Fig. S1C-E), confirming that the single-copy gene insertion method indeed reduces tau expression and that tau expression level can contribute to phenotype. Finally, a strain where a small 15 amino acid V5-epitope tag was substituted for Dendra2 in the TauT4 transgene exhibited a similar phenotypic deficit as the TauT4 overexpression strain, suggesting that the deficit is caused by tau and not by the fusion of Dendra2 to tau (Fig. S1B).

In order to address the effect of tau PTM-mimetics, CRISPR-Cas9 gene editing [48, 49] was used to introduce phosphomimetic T231E, phosphoablation T231A, and acetylmimetic K274/281Q mutations into the TauT4 ORF (Fig. 2a, b). For simplicity, these tau PTMmimetic and phosphoablation mutants will be referred to as T231E, T231A and K274/281Q. Our results clearly demonstrate that T231E exhibited subtle but significant defects in touch responsiveness at both day 3 and day 10 , while K274/281Q was different from the Dendra2 control only at day 10 (Fig. 2c, d). However, between day 3 and day 10, the touch sensitive phenotype of K274/ 281Q worsened significantly. This may indicate either a ceiling effect of T231E or a sensitized K274/281Q progression with age. In contrast, T231A was indistinguishable from TauT4 (Fig. 2c, d).
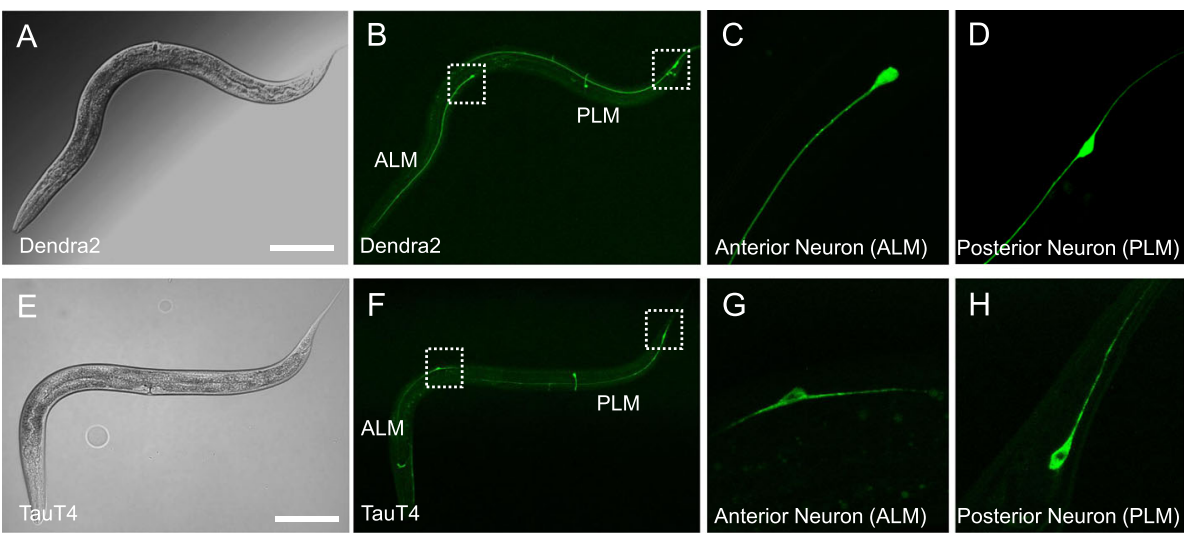

Fig. 1 Expression of Dendra2 and TauT4 from a single-copy transgene in C. elegans touch neurons. DIC $(\mathbf{a}, \mathbf{e})$ and confocal fluorescent images (b, c, d, $\mathbf{f}, \mathbf{g}$, and $\mathbf{h}$ ) are shown of $L 4$ larval worms expressing single-copy transgenes coding for Dendra2 (b, $\mathbf{c}, \mathbf{d}$ ) or a Dendra2::TauT4 translational fusion (f, $\mathbf{g}$, h). The transgenes are driven by the mec-7 promoter in ALM(L/R) and PLM(L/R) neuron pairs, and also in AVM and PVM neurons, which are not considered further here. Panels $\mathbf{c}, \mathbf{g}$ and $\mathbf{d}, \mathbf{h}$ are magnifications of ALM and PLM respectively in the areas encompassed by the white boxes in panels (b and f). Scale bars: $25 \mu \mathrm{m}$. ALM is Anterior Lateral Microtubule and PLM is Posterior Lateral Microtubule cells, mechanosensory neurons that mediate behavioral responses to light touch to the bödy wall within the receptive fields definèd by their projections 


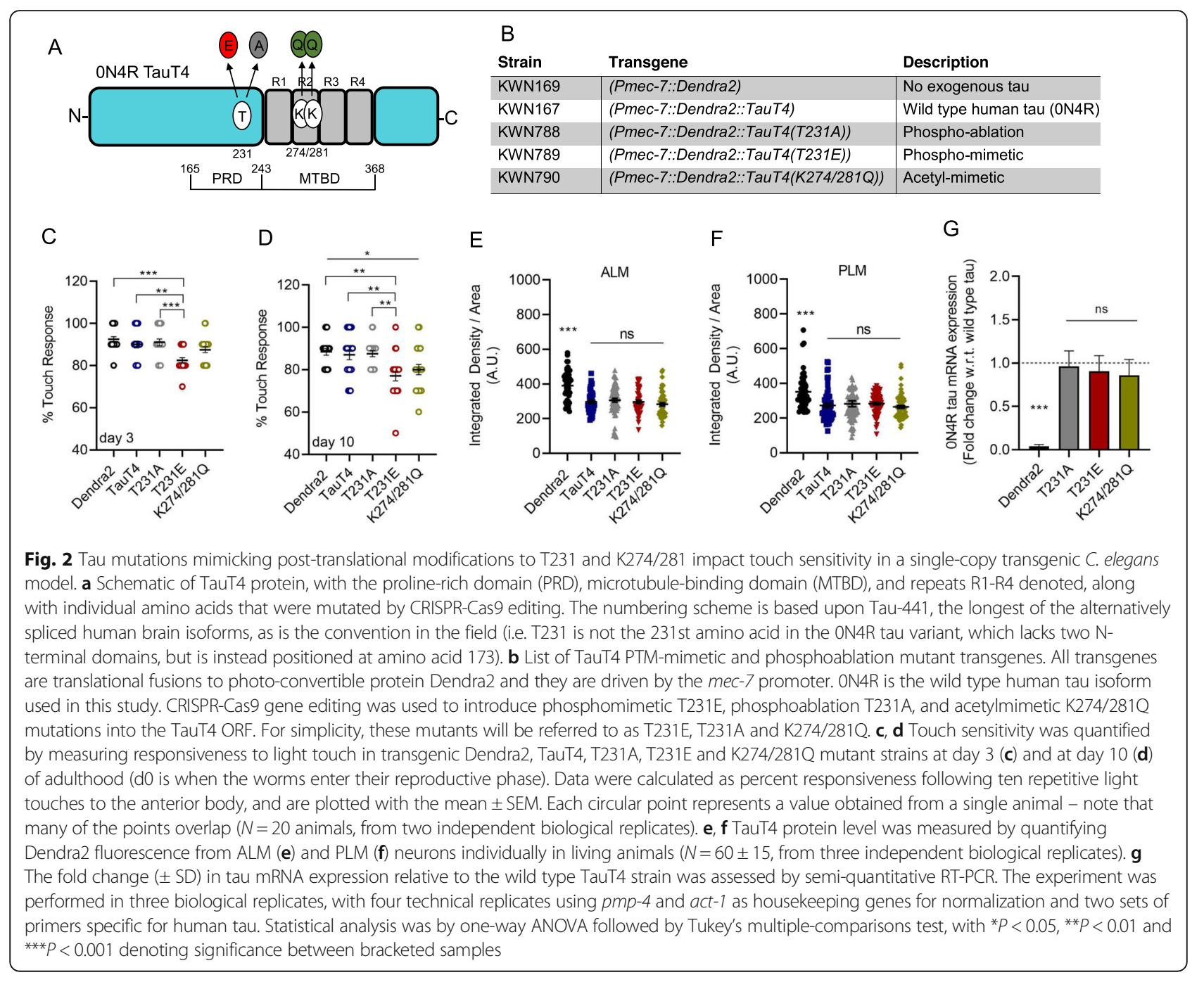

We also evaluated several other stereotypical behavioral measures that have been shown to be influenced by age but do not involve touch cell neurons, including thrashing in liquid (Fig. S2A, B) and basal locomotion on solid media (Fig. S2C, D). Taken together, these data suggest that the effect of pathological, disease-relevant tau expression in touch sensory neurons is restricted to the behavioral response to light touch.

Differences in tau expression levels are unlikely to be responsible for the differences in touch sensitivity between strains, as assessed by two independent measures: first, quantifying TauT4 protein fluorescence in both the pairs of neurons (ALM and PLM) (Fig. 2e, f) and second, measuring tau mRNA levels (Fig. 2g). We note, however, that Dendra2 exhibits slightly greater fluorescence alone than when fused to tau, for unknown reasons (Fig. 2e, f). Moreover, since survival plots of the various strains used in this work were statistically indistinguishable, we were able to rule out any phenotypic age-dependence being due to a change in lifespan (Fig. S2E, F). These novel observations represent a first-in-kind platform for studying cryptic tau phenotypes and physiologic consequences in the absence of overt baseline defects.

\section{T231E and K274/281Q mutants cause age-dependent abnormalities in neurite morphology}

Normally, touch neurons are organized into precise anterior and posterior receptive fields, defined by the physical architecture of sensory neurites from $\operatorname{ALM}(\mathrm{L} / \mathrm{R})$ and $\operatorname{PLM}(\mathrm{L} / \mathrm{R})$; these neurites extend along the anterior or posterior half of the body, respectively, but do not overlap [59]. Aging phenotypes in touch receptor neurons include a low incidence of morphologic defects, such as increased neurite overlap due to an overextension defect [60]. We investigated whether single-copy expression of Dendra2, TauT4 or the PTM-mimetic and phosphoablation mutants exacerbated these defects. A transgene consisting of an integrated Pmec-4::mCherry expression cassette was used to visualize the touch neurons using confocal 
microscopy (Fig. 3a-d, S3A, B). We found that T231E strongly and significantly increased the incidence of overextension from $\sim 4 \%$ to $\sim 40 \%$ by day 3 of adulthood (Fig. 3e). However, the TauT4 and K274/281Q mutants were not significantly different from Dendra 2 controls in day 3 adults (Fig. 3e).

In addition to the overextension defect, other neuritic abnormalities developed with age, such as branching, guidance defects, beading, and breakage (Fig. 3c, d, S3A, $\mathrm{B})$. While none of the strains were significantly different in terms of these defects at day 3 (data not shown), both T231E and K274/281Q exhibited an increased incidence of overextension, misguidance, and gap defects at day 10 , but were not different with respect to branching or beading compared to the Dendra2, TauT4 or T231A mutant strains (Fig. 3f-h, S3C, D). It was intriguing that age exacerbated the overlap defect in K274/281Q ( $p=$ 0.05 , between day 3 and day 10), which mirrored its effect on touch sensitivity, but that T231E had reached its maximum penetrance by day 3 of adulthood. These results suggested to us that this model is appropriate to detect subtle differences in pathology and "disease" progression as a function of specific tau PTM mimetics.

\section{Mitochondrial fragmentation in tau PTM-mimetic mutants}

Impaired mitochondrial dynamics and excessive fragmentation have been observed in $\mathrm{AD}$ postmortem brains and in AD mouse models [66-68]. However, the effects of disease-relevant, site-specific phosphorylated or acetylated tau on mitochondrial morphology in neurons have not been thoroughly studied in the absence of tau overexpression. To investigate whether a causal relationship exists between tau PTM-mimetics and mitochondrial morphology, we examined the mitochondrial network in the PLM cell bodies at day 3 and at day 10 of adulthood in our tau PTM mutant models. Touch cell mitochondria were labeled with mitomKeima, a pH-sensitive fluorescent biosensor [61, 62]. Mito-mKeima can be used as a dual excitation ratiometric mitophagy reporter, as we expand upon below (Figs. 5 and 6). However, here we used single wavelength excitation-emission imaging of mito-mKeima in the appropriate channel to visualize mitochondrial structure, such as shown in Fig. 4. Under these image acquisition conditions, mitochondria are visible, but mitochondria that have been engulfed by acidic vesicles are not (for convenience, heretofore we will refer to these structures as "mitolysosomes"). Based upon these images, mitochondria were categorized into four levels, from normal tubular-reticular morphology through increasing degrees of fragmentation (Fig. 4a-d and Methods). Neuronal mitochondria from day 3 adult animals had generally tubular-reticular morphology, and their distribution was independent of tau genotype (data not shown). However, by day 10 of adulthood, all of the strains contained some fragmented mitochondria, consistent with age-associated remodeling, but it was clear that T231E and K274/281Q were significantly more fragmented than Dendra2, TauT4 or T231A (Fig. 4e).

\section{Mutations mimicking pathologic tau modifications suppress stress-induced mitophagy}

Next, we employed mito-mKeima in dual excitation mode in order to assess organelle turnover. Throughout a neuron's lifetime, aged and damaged mitochondria undergo dynamic recycling and elimination [69]. Mitophagy is a type of cargo-selective autophagy where defective mitochondria are engulfed by mitophagosomes and subsequently degraded by fusion with mitolysosomes (Fig. S4) [70]. This process of mitochondrial quality control $(\mathrm{MQC})$ can be impaired during aging and has been associated with major neurodegenerative disorders including $\mathrm{AD}$ [71, 72]. Mito-mKeima has a unique spectral characteristic whereby at neutral $\mathrm{pH}$ or above, such as occurs in the mitochondrial matrix, excitation at $440-\mathrm{nm}$ results in emission at $600-\mathrm{nm}+$, but at acidic $\mathrm{pH}$, such as occurs in the lysosome, the excitation maxima shifts to 550-nm (akin to a conventional red fluorescent protein) (Fig. 5a-f). Mito-mKeima is also resistant to degradation by lysosomal proteases. These characteristics allows a mitophagy index to be calculated using dual excitation ratio imaging that reflects the relative amount of mitochondria that have undergone engulfment and fusion with acidic vesicles (Fig. $5 \mathrm{~g}$, i). In addition, because these mitolysosomes are spectrally and morphologically distinct, we can also assess their absolute abundance and size (Fig. 5h, j).

In PLM neurons, pathologic tau PTM-mimetic mutations T231E and K274/281Q had little effect on baseline mitophagy, but decreased the number of mitolysosomes in young adults (Fig. 5h). We also note an apparent increase in the mitophagy index and reduction in the number of mitolysosomes with age that reached significance in Dendra2, TauT4, and T231A, but not in T231E and K274/281Q (Fig. 5g-j).

Mitophagy is a selective form of macroautophagy (referred heretofore as autophagy). To address whether the differences we observed with the T231E and K274/ 281Q mutants were restricted to mitophagy or were reflected in general autophagy, we expressed a tandemtagged mCherry-GFP-Atg8/LGG-1 reporter [73, 74] specifically in mechanosensory neurons. We found no significant differences in the number of autophagosomes (APs) (green puncta) or autolysosomes (ALs) (red puncta due to GFP fluorescence quenching in the acidic autolysosome environment) among our strains at either day 3 or day 10 (Fig. S5). In addition, ALs were more abundant than APs in all transgenic animals at both the 

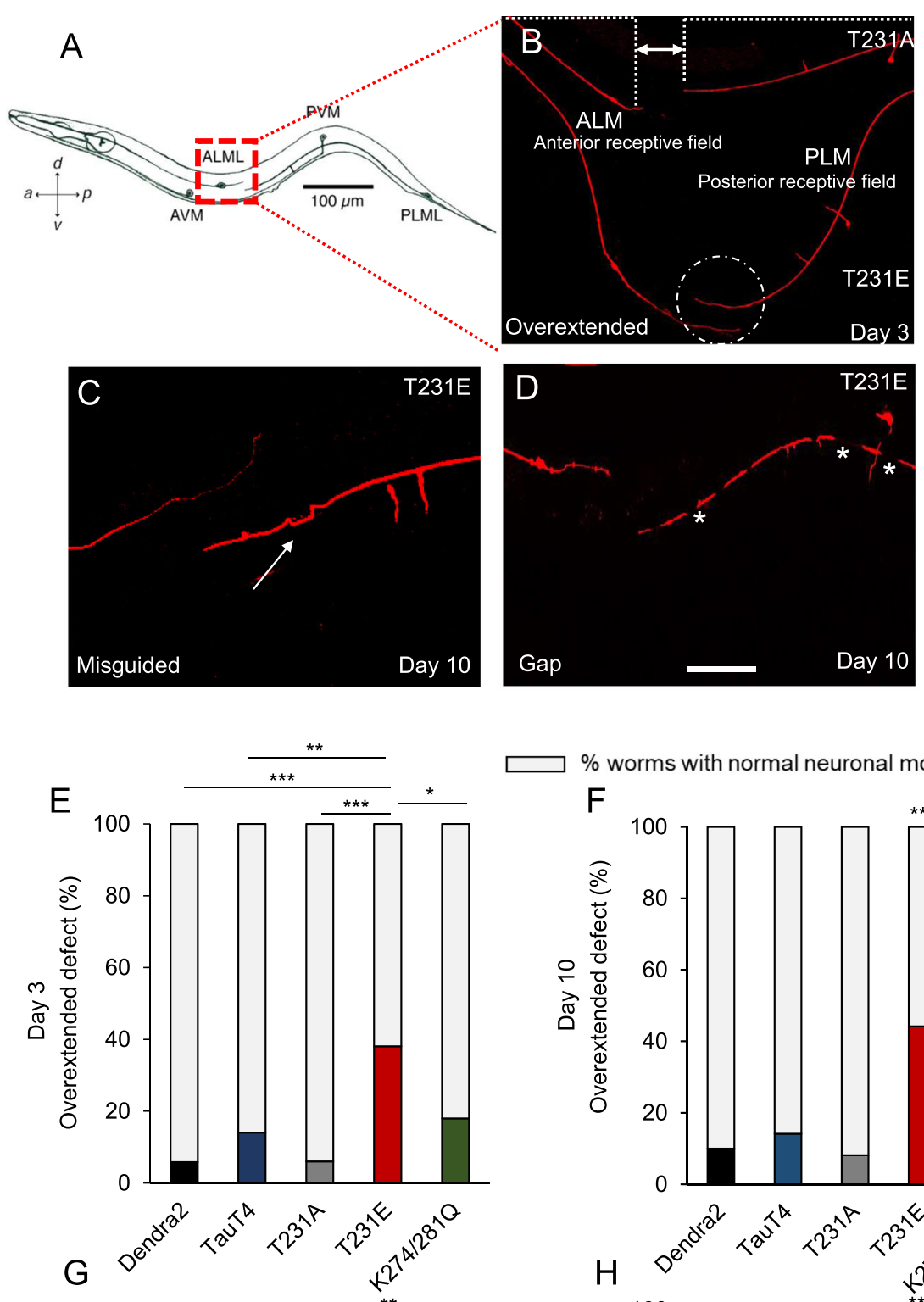

$\%$ worms with normal neuronal morphology
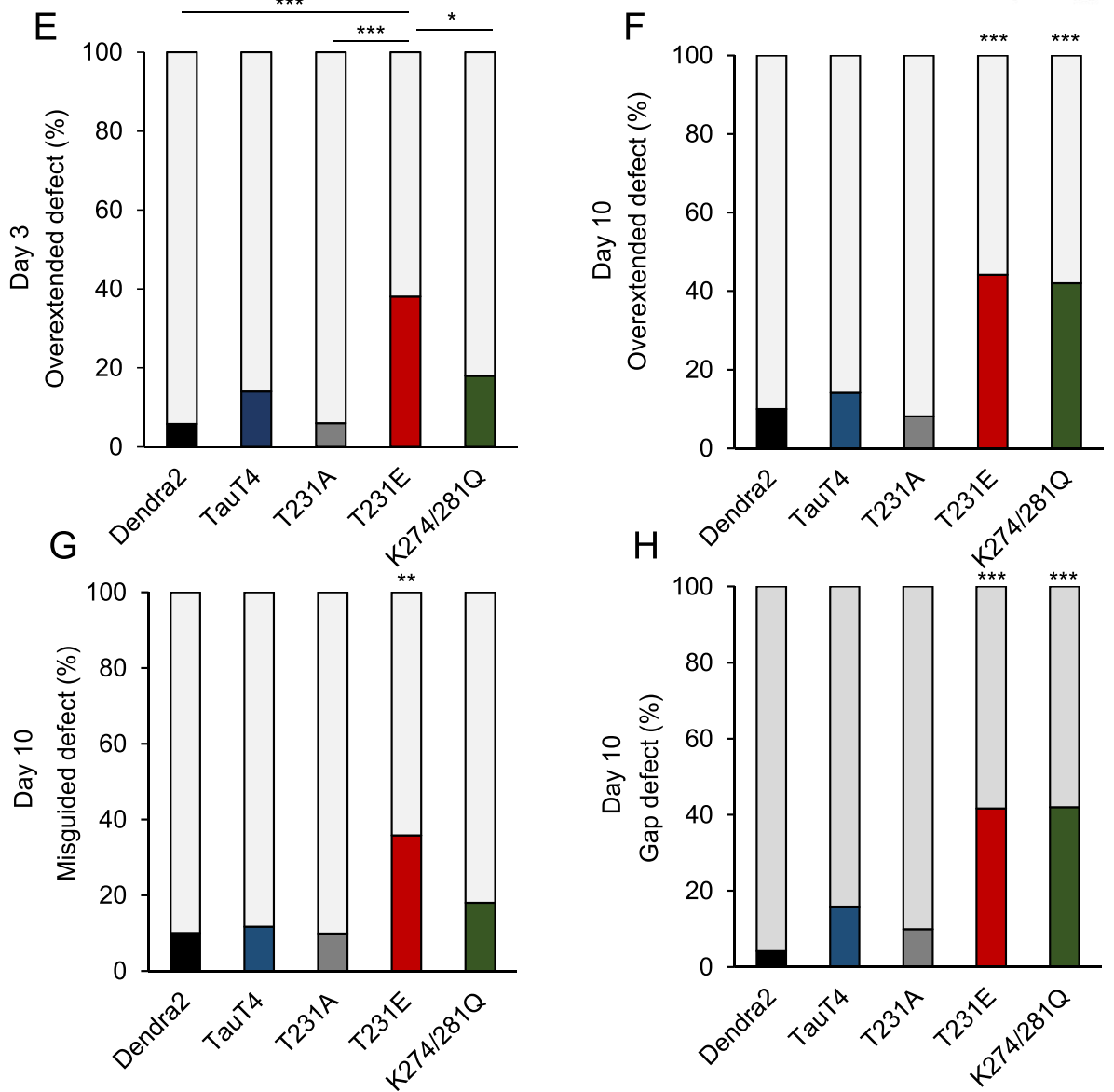

Fig. 3 (See legend on next page.) 
(See figure on previous page.)

Fig. 3 Strains with tau mutations mimicking post-translational modifications to T231 and K274/281 display abnormal touch receptor neurite morphology. a Schematic of a hermaphrodite animal. Mechanosensory neurons pairs ALM(R/L) and PLM(R/L) are present on both left and right sides of the animal, but only one of each pair is depicted. In wild type animals, neurites projecting from ALM and PLM do not overlap with each other, but instead divide the animal's body into two distinct receptive fields, as indicated (modified from 79). b Neurons were visualized using a Pmec-4::mCherry fluorescent reporter. Two animals lying side-by-side are shown here. The top animal is from the phospho-null strain (T231A) and the bottom animal is from the phospho-mimetic strain (T231E). The normal separation between the ALM and PLM neurites, represented by the area between the dashed lines in T231A, is replaced by overlapping neurites in T231E, as demarcated by a dashed circle. c, $\mathbf{d}$ Representative images of specific neurite morphology defects observed in touch cells. White arrow points to a misguided neurite in panel (c), and white stars illustrate gaps in panel (d), respectively. The scale bar is $10 \mu \mathrm{m}$. e-h Quantification of the defects exemplified in panels $\mathbf{b}-\mathbf{d}$ in Dendra2, TauT4, and T231A, T231E and K274/281Q. The colored bar denote the percentage of worms with the defect, while the gray bar denotes the percentage of worm that lack the defect. Statistical analysis was by Fisher's exact test followed by two-tailed correction, with ${ }^{*} P<0.05$ compared to the Dendra2 control, unless otherwise denoted. Data for the parental Pmec-4::mCherry reporter strain lacking tau transgenes, which is very similar to Dendra2, is not shown. $N=50$ neurites from separate animals scored for each type of defect, from three independent biological replicates

days observed (Fig. S3F-I), suggesting that turnover of APs (i.e. formation of APs and conversion to ALs) is faster than turnover of ALs. Taken together, these experiments demonstrate that while general autophagy is similar in all the strains, the T231E and K274/281Q strains exhibit subtle alteration in mitophagy, particularly with age (Fig. 5i).

Next, we sought to evaluate the impact of oxidative stress on neuronal mitophagy. These studies are particularly significant, as chronic mitochondrial stress is likely to be a factor in neurodegenerative diseases including $\mathrm{AD}$ [75]. To induce mitophagy, Dendra2, TauT4 and PTM-mimetic mutant strains expressing mito-mKeima in touch cells were treated with $8 \mathrm{mM}$ mitochondrial complex I inhibitor paraquat (PQT) overnight. PQT has been used extensively in worms, including for this purpose [56, 57]. The next day, mitophagy was assessed through dual-excitation ratio imaging. Unsurprisingly, PQT treatment increased mitophagy in Dendra2 at both day 3 and day 10 of adulthood (Fig. 6e, f). As found for previous measures, TauT4 (Fig. 6a, b) and T231A were indistinguishable from Dendra2 (Fig. 6e, f). However, PQTinduced mitophagy was abolished in T231E and K274/281Q at both day 3 and day 10 (compare Fig. $6 \mathrm{~b}$ and $\mathrm{d}$ as well as e, f). Finally, we note that PQT does not induce bulk autophagy in PLM neurons in any of our strains (data not shown). We conclude that mutations that mimic site-specific phosphorylation and/or acetylation of tau, in addition to being a mitocentric stress in-and-of themselves, have the ability to reduce normal mitochondrial responses to subsequent stress, which could impact mitochondrial function and neuronal health during aging.
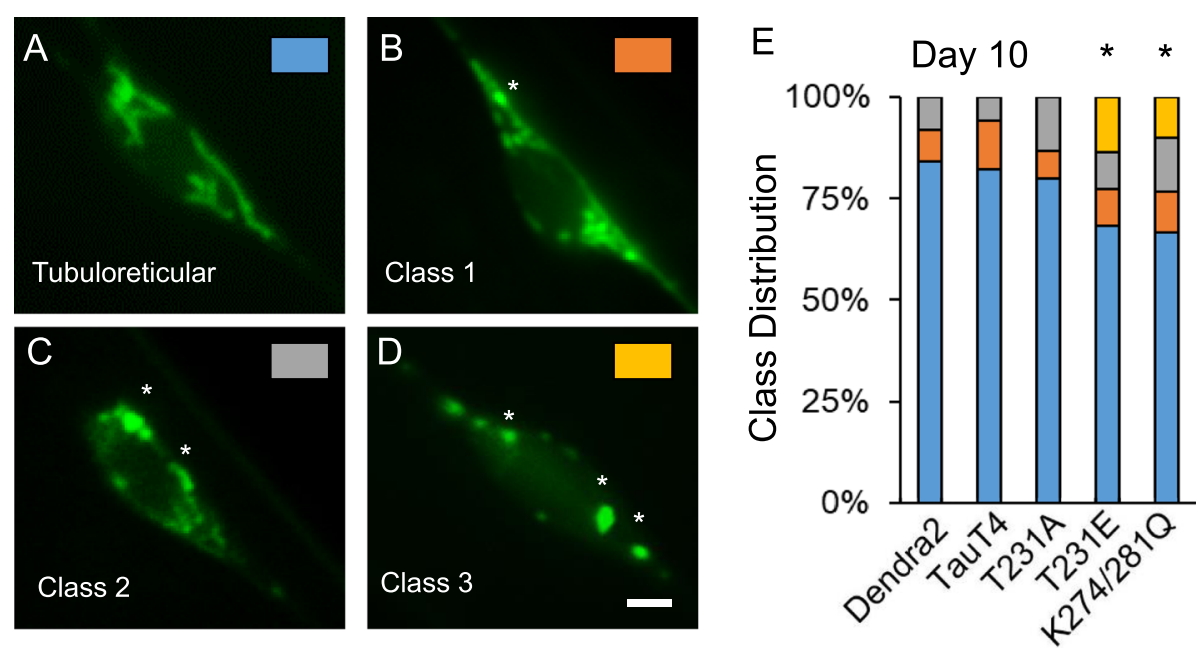

Fig. 4 Tau mutations mimicking post-translational modifications to T231 and K274/281 cause mitochondrial fragmentation. a-d Representative images of mitochondria from PLM neuron cell bodies showing different classes of fragmentation. Each panel is color-coded to the data in panel (e), as indicated. Asterisks denote overt swollen mitochondria resulting from excessive fragmentation. Labeling was via mito-mKeima, imaged on a single channel specific for mitochondria. e Data from day 10 adults presented in a more granular fashion, with individual cells assigned a category as depicted in panels (a-d). $N=30 \pm 5$ cells from separate animals, performed in two independent biological replicates. Statistical analysis was by Wilcoxon signed-rank test, with ${ }^{*} P<0.05$ compared to Dendra2 

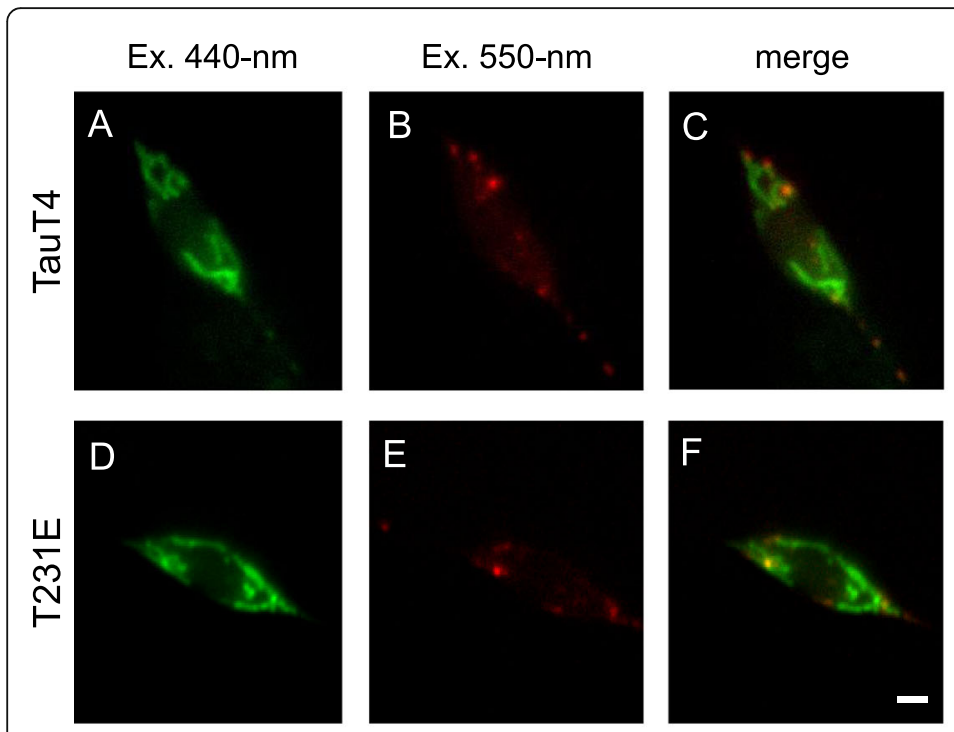
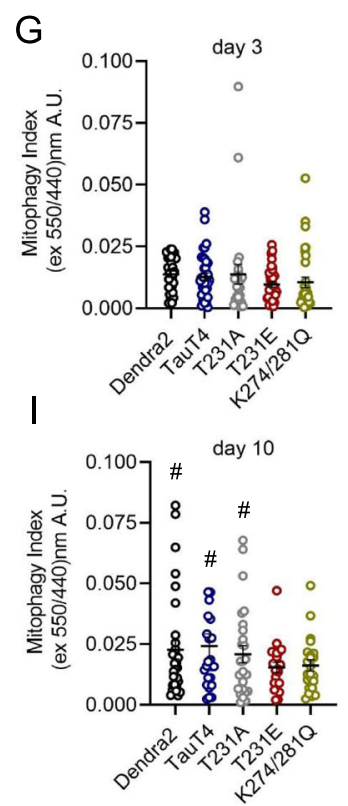
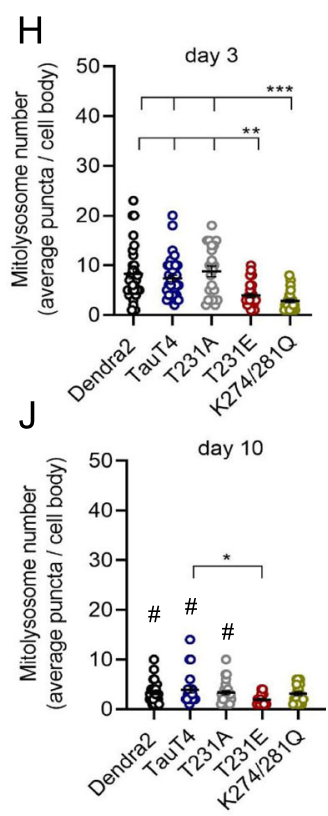

Fig. 5 Tau mutations mimicking post-translational modifications to T231 and K274/281 reduce the number of mitolysosomes, but not baseline mitophagy. a-f Representative fluorescent images from the PLM cell bodies expressing single-copy TauT4 or T231E, together with the biosensor mito-mKeima. Mitochondria at neutral pH have been pseudo-colored green, and organelles that have been incorporated via mitophagy into acidic vesicles have been pseudo colored red. Scale bars: $5 \mu \mathrm{m}$. g, i Background corrected 550-nm excitation / 600-nm emission values were divided by 440-nm excitation / 600-nm emission values to obtain a mitophagy index for PLM cell bodies from Dendra2, TauT4, and T231A, T231E, and K274/281Q PTM mutants at day 3 and day 10 of adulthood. $\mathbf{h}, \mathbf{j}$ Quantitative analysis of the number of mitolysosomes containing mitochondria in the distal PLM cell bodies of day 3 and day 10 adult animals as a function of tau genotype, as indicated. Data are the mean \pm SEM from three independent technical replicates performed on different days. Individual data points demarcate values from single PLM cells from separate animals $(N=35 \pm 5)$. Statistical analysis within day 3 and day 10 datasets was by one-way ANOVA with Tukey's multiple comparison test, with ${ }^{* *} P<0.001$, ${ }^{*} P<0.01,{ }^{*} P<0.05$ when comparing bracketed samples. Comparisons between day 3 with day 10 data were limited to within a single genotype, and significance was determined by Student's t-test, with ${ }^{\#} P<0.05$

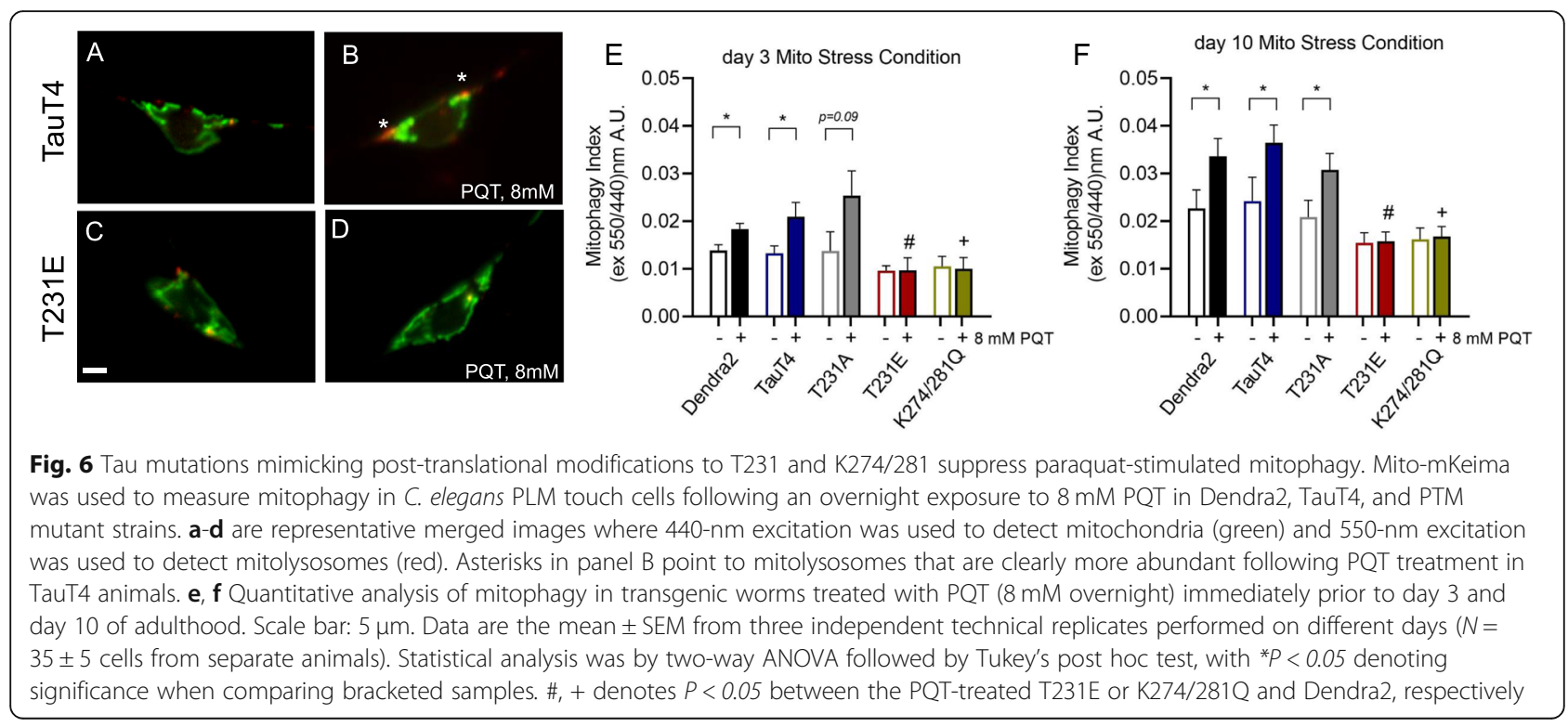




\section{Discussion}

A characteristic hallmark of the $\mathrm{AD}$ brain is the presence of tau with PTMs defined as pathological, that likely contribute to the onset and progression of the disease. Phosphorylation of tau at specific epitopes is widely appreciated to contribute to $\mathrm{AD}[7,9]$, with acetylation of tau at specific sites also shown to contribute to the evolution of tau pathology $[19,20]$. While it has now become evident that the insoluble accumulations of tau in the AD are likely not the primary toxic species $[5,7,8,76]$, the specific mechanisms by which monomers or soluble oligomers of tau with disease-relevant PTMs cause neuronal dysfunction have not been full delineated. This is due in part to the fact that the majority of studies have used models in which tau is overexpressed, which can result in outcomes that may not be directly relevant to AD pathogenesis [33, 38, 40]. Since Alzheimer's disease progresses with age [77], we need a viable strain that is healthy over a long period of time, unlike the available overexpression strains which are severely short-lived and thus conduction of phenotypic or behavioral assays are extremely difficult at later time point of a C. elegans life cycle [40].

To avoid this potential confounding factor, we generated a $C$. elegans model containing single-copy expression cassettes coding for tau and tau with diseaseassociated PTM-mimetic mutations, where a short lifespan and easy-to-use genetic tools contribute to monitoring the progression of pathology as function of age and its link with mitochondrial abnormalities. Our lab generated single-copy tau model is different from other available tau overexpression $C$. elegans models as the strain expressing wildtype tau lacks overt abnormalities, including in health or lifespan, and baseline mitophagy is not impaired $[39,78]$. However, a key limitation associated with this model is the challenge of biochemical assays. Since tau is expressed at single-copy levels in just the six touch neurons, specific solubility assays using these transgenic strains are not feasible. Combined with the presence of an external cuticle, the same is true for detecting thioflavin+ tau aggregates. Despite this drawback, our model has led to new insight into a mechanism by which tau with mutations that mimic disease-relevant PTMs impairs neuron function, and the reduced expression level has allowed us to describe a cryptic phenotype that may have otherwise been obscured.

Although 0N4R human brain tau isoform contains almost 70 potential phosphorylation sites that span the entire molecule [4], only select residues are phosphorylated physiologically and/or pathologically. One key diseaserelevant site is T231 that shows increased phosphorylation early in the evolution of AD tau pathology and greater levels in "pre-tangle" neurons [9]. Phosphorylation of T231 results in a decrease in microtubule association [79], likely due to the conformational shift and decreased tubulin binding that was observed with a pseudophosphorylated tau construct [11]. A S235/T231E tau construct also showed mislocalization in mature neurons [80]. Intriguingly, we observed that at day 3 worms expressing T231E showed subtle but significant defects in touch sensitivity and neurite morphology, while those expressing the acetylation mimic K274/281Q did not. However, by day 10 significant deficits in touch sensitivity and neurite morphology were observed in both T231E and K274/281Q. Thus functional decline of the touch neurons due to tau modifications is highly correlated with altered neuron morphology providing hints towards commonalities with the aging mammalian brain and suggesting conserved mechanisms can be operative in neuronal decline across phyla [81]. However, it is as-of-yet unclear why certain neuronal defects (such as over extension, misguidance and gap) predominate. This could be particularly interesting in the context of neuronal regeneration. Overall these data may suggest that phosphorylation at T231 is an early initiator of tau dysfunction in AD. These findings also correlate with the fact that increases in phosphorylation at T231 precede increased acetylation at K274/281 in the evolution of $\mathrm{AD}$ tau pathology $[4,82]$.

Mitochondria are crucial metabolic hubs dictating cell fate decisions, and mitochondrial dysfunction likely plays a critical role in the pathogenesis of $\mathrm{AD}[24,25$, 81]. Mitochondria possess dedicated MQC mechanisms to ensure their fidelity [70]. Abnormalities in MQC pathways noted to occur in $\mathrm{AD}$ [69] may arise in part through the action of tau species with aberrant PTMs [83]. Mitophagy, which is a form of selective autophagy that delivers dysfunctional mitochondria to lysosomes for recycling, is a key player in MQC $[69,84]$. C. elegans have been widely used to study neuronal function, aging, and MQC mechanisms, as well as to model proteotoxic neurodegenerative disorders [85]. Therefore, we next examined the impact of $\mathrm{T} 231 \mathrm{E}$ and $\mathrm{K} 274 / 281 \mathrm{Q}$ on mitochondrial biology. In contrast to the deficits in touch sensitivity and neuronal morphology observed at day 3, neither T231E nor K274/281Q negatively impacted mitochondrial morphology at that age. However, by day 10 mitochondrial fragmentation was significantly exacerbated in T231E and K274/281Q, which could be reflective of a deficit in mitophagy. Therefore, we measured the relative amount of mitochondria that were engulfed and fused with acidic compartments, as well as the absolute abundance of mitochondria in acidic compartments ("mitolysosomes").

Interestingly, we found that in control animals, the mitophagy index, a measure of relative mitolysosome to mitochondria abundance, increased with age, and the number of mitolysosomes decreased (Fig. 5). The factors critical for the effective turnover of damaged mitochondria during aging likely include underlying stress, as well as autophagic and lysosomal capacities. While there are other 
reports of mitophagy increasing with age in systems including Drosophila [86], mouse [87] and human disease [88], there is also evidence that mitophagy becomes, like many types of stress responses, impaired with age [89]. Although our data support the former studies, we note that mito-mKeima is resistant to acid proteases and likely accumulates over time. In fact, a decreased number of brighter mitolysosomes in day 10 animals may represent cumulative vesicle fusion, and so we need to temper our conclusion to reflect this caveat. Nevertheless, we were able to stimulate mitophagy using PQT at day 10 to a similar extent as day 3 (Fig. 6), confirming that, at a minimum, the ability to generate a robust response to oxidative stress is maintained in older wild type animals.

Our results demonstrate a striking abolition of PQTinduced mitophagy in the tauopathy-relevant T231E and K274/281Q mutants (Fig. 6). This observation is consistent with defective mitophagy being a prominent feature in age-related disorders [90], including AD [91, 92], and contributing to premature aging such as observed in Werner's syndrome patients and invertebrate Werner's disease models [93]. It is also particularly intriguing that the T231E and K274/281Q do not appear to exhibit the same age-dependence as Dendra2, TauT4, or T231A. This could be interpreted to mean that these mutants exhibit characteristics that appear in older adults. Their inability to response appropriately to oxidative stress at both a young and old age - suggests that the mitochondria in fact do have baseline defects, albeit at a level that is not discernable in the absence of stress. The recent finding that mitophagy enhancement can suppress AD-related phenotypes in tau transgenic animals lends support to this idea [39].

Studies in living AD patients and postmortem brain tissue have provided evidence that in affected brain regions there is impaired mitochondrial function and an accumulation of unrepaired damaged mitochondria [84, 94]. Further, there are data indicating that pathological tau species may impair mitophagy, however the precise mechanism of tau induced mitophagy impairment remains to be delineated. It has been demonstrated that both human wild type tau (hTau) and FTLD tau (P301L) inhibited mitophagy in neuroblastoma cells, by reducing mitochondrial translocation of Parkin [95]. Tau specifically impaired Parkin recruitment to defective mitochondria by sequestering it in the cytosol. This sequestration was mediated by aberrant interactions of Parkin with the projection domain of Tau [95]. Future questions regarding mechanism and signaling pathway will undoubtedly be of great interest to the scientific research community and are underway.

Moreover, it will be of interest to determine whether the tau mutants described here are perceived as stressors, and hence cause activation of a retrograde response, such as has been described previously for the C. elegans Nrf2 ortholog SKN-1 in adaptation to a decrease in mitophagy [96]. Alternatively, other retrograde signaling pathways such as that mediated by ATFS- 1 and the mitochondrial unfolded protein response (mtUPR) can also mediate adaptation to mitochondrial stress [97], including stress due to defects in mitophagy machinery [98]. However, prolonged cellular activation of the mtUPR has been shown to be maladaptive in a C. elegans model of dopaminergic neurodegeneration [99], suggesting that ultimate role of stress response pathways is context dependent. It is also possible that the single-copy tau mutants do not elicit stress-responses in-and-of themselves, but instead sensitize neurons to additional stressors, consistent with our mitophagy results.

\section{Conclusion}

In conclusion, to our knowledge this is the first study to clearly demonstrate that single-copy expression of tau with Tauopathy associated PTM-mimetics - mutations that mimic pathologic PTMs of tau - impair neuronal function and structure in an age-dependent manner. One advantage of our single-copy model is that it allows us to quantitatively measure subtle deficits and discriminate between the effects of mutations that mimic distinct PTMs. For example, we demonstrate that T231E presents with a neuronal functional (and morphological) deficit earlier than K274/281Q. Since stress-induced mitophagy was abolished equally by both, it is likely that distinct forms of Tauopathy associated, pathologic tau may differentially impact neuron structure/function through at least one other mechanism. However, further studies are needed to determine if these pathways are separate and isolated, and if they interact, whether they are additive or synergistic. We anticipate that this new C. elegans AD model represents a foundation to achieve a more nuanced understanding of how pathologic tau impacts neuronal function.

\section{Supplementary information}

The online version contains supplementary material available at https://doi. org/10.1186/s13024-020-00410-7.

\footnotetext{
Additional file 1: Figure S1. Touch responsiveness controls and defects associated with tau overexpression. (A) Touch assays were conducted on day 3 and day 10 adult animals grown at $20^{\circ} \mathrm{C}$ using glr1 (n2461) and mec-4(U253) strains as negative controls. The data are the mean \pm SEM ( $N=10-30$ animals, from three independent replicates). Statistical analysis was by one-way ANOVA followed by Tukey's multiplecomparisons test with ${ }^{*} P<0.001$ denoting significance between bracketed samples (ns is not significant). Each point represents a value obtained from a single animal, which was touched 10 times, with a $10 \mathrm{~s}$ gap between each touch. (B) Bar graphs represent the average percent touch response of the overexpressed human tau, fused with Dendra2 or V5. Experiment was performed on day 3 and day 10 adult animals with $N=15-20$. All the $p$-values are represented as ${ }^{*} p<0.05,{ }^{* *} p<0.01$ and ${ }^{* * *} p<0.001$. Both data are from two biological replicates. (C-E)
} 
Representative images (40x magnification) of a PLM neuron in: (C) the MosSCl single-copy integrated tau strain (50 ms exposure), (D) a multicopy array tau strain (5 ms exposure), and (E) a $50 \mathrm{~ms}$ exposure of the multi-copy strain, suggesting a 10-fold difference in relative expression level. (DOCX 367 kb)

Additional file 2: Figure S2. Thrashing, locomotion, and lifespan are unaltered in strains with Tau mutations mimicking post-translational modifications to T231 and K274/281. Dendra2, TauT4, T231A, T231E and K274/281Q PTM mutant strains at day 3 and at day 10 of adulthood were assessed for (A, B) thrashing behavior in liquid media, (C, D) locomotion on solid media measured as the rate of body bends, (E) survival, or (F) lifespan. The data are the mean \pm SEM. ( $N=20$-to-50 worms per genotype from three independent biological replicates, with each data point in $A, B$ representing an individual worm). Statistical analyses were by one-way ANOVA or Mantel-Cox/ log-rank analysis. No statistical differences were found between genotypes for any of the three measures (denoted ns). (DOCX $313 \mathrm{~kb}$ )

Additional file 3: Figure S3. Abnormal touch receptor neurite morphology in the older animals. Representative images of specific neurite morphology defects observed in touch cells, especially in day 10 adult animals. White stars illustrate beads or pearl like structures in panel $A$ and dashed circle denote branching in panel $B$. The scale bar in panel B is $10 \mu \mathrm{m}$. (C, D) Quantification of the defects exemplified in panels A, B in Dendra2, TauT4, and T231A, T231E and K274/281Q. The colored bar denote the percentage of worms with the defect, while the gray bar denotes the percentage of worm that lack the defect. Statistical analysis was by Fisher's exact test followed by two-tailed correction, with ns denotes statistically not significant. Data for the parental Pmec-4::mCherry reporter strain lacking tau transgenes, which is very similar to Dendra2, is not shown. $N=50$ neurites from separate animals scored for each type of defect, from three independent biological replicates. (DOCX $143 \mathrm{~kb}$ )

Additional file 4: Figure S4. Schematic representation of using mitomKeima to measure mitophagy. Healthy mitochondria have a matrix $\mathrm{pH}>7$ (green) whereas dysfunctional mitochondria undergoing mitophagy and engulfed by lysosome are exposed to an acidic $\mathrm{pH}<5$ (red). Mito-mKeima is an acid-protease resistant fluorescent protein whose excitation maxima shifts from 440-nm to 550-nm with acidification. By measuring the ratio of emissions at $600-\mathrm{nm}$ following sequential excitations at the two excitation maxima, you can obtain an estimate of the relative amount of mitochondria that are in neutral compared to acidic environments. In addition to spectral differences, mitochondria that have been engulfed by autophagosomes (labeled mitolysosomes) are generally round rather than tubuloreticular, and hence morphology can generally be used as a second measure to distinguish between organelles. However, this difference can be masked by mitochondrial fragmentation. (DOCX $140 \mathrm{~kb}$ )

Additional file 5: Figure S5. Strains with tau mutations mimicking post-translational modifications to T231 and K274/281 have normal levels of baseline autophagy. (A-E) Representative fluorescent images from the PLM cell bodies expressing single-copy TauT4 or PTM-mimetics, together with an $m$ Cherry::gfp:.Igg-1 reporter. Please note that the reporter fluorescence far exceeds and overwhelms that of the Dendra2 fusion. Autophagosome (AP) at neutral pH have been pseudo-colored green, and autolysosome (AL) have been pseudo-colored red. Scale bar $=100 \mu \mathrm{m}$. Note that the intensity of red fluorescence coming from distinct red AL compared to green of AP is stronger; thus the gain of red channel was purposefully set lower for all the images taken at Nikon Confocal microscope (see materials and methods) Quantification of APs and ALs in the PLM neurons of Day $3(\mathrm{~F}, \mathrm{G})$ and Day 10 (H, I) Dendra2, TauT4, T231A, T231E and K274/281Q PTM mimetic strains. Data are the mean \pm SD of $\geq 30$ animals combined from two independent biological replicates. Data for the parental LGG-1 reporter strain lacking tau transgenes, which is very similar to Dendra2, is not shown. (DOCX $462 \mathrm{~kb}$ )

Additional file 6: Table S1. Detailed quantification of the neuronal defects observed in day 10 transgenic worms. The most significant defects observed in the strains with tau mutations mimicking posttranslational modifications to T231 and K274/281 are the overextension of ALM/PLM and gaps or breaks observed in their neuronal processes. (DOCX $33.2 \mathrm{~kb}$ )

\section{Abbreviations}

AD: Alzheimer's disease; CRISPR: Clustered regularly interspaced short palindromic repeats; Cas9: CRISPR associated protein 9; ETC: Electron transport chain; MQC: Mitochondrial quality control; MosSCI: Mos-mediated single copy insertion; NGM: Nematode growth media; PQT: Paraquat; PTM: Post-translational modification

\section{Acknowledgements}

Technical assistance provided by Anson Cheng, Teresa Sherman, Yunki Im, Joseph Cartella, Alan Alberto, Brian Jones and Ejiroghene Okarevu was greatly appreciated. We thank the members of Dr. Johnson's lab, the Mitochondrial Research and Interest Group at the University of Rochester Medical Center and the members of the Western New York worm meeting for their valuable suggestions and helpful discussions, and Drs. Christopher Rongo and Euchan Park for sharing a worm codon optimized version of mito-mKeima prior to publication.

\section{Authors' contributions}

Conceived and designed the experiments: SG GJ KN. Performed the experiments: SG SF. Analyzed the data: SG SF GJ KN. Wrote the paper: SG GJ $\mathrm{KN}$. All authors read and approved the final manuscript.

\section{Funding}

This work was supported by NIH (R21AG060627 and R01AG067617) (KN and GJ). Some strains were provided by the CGC, which is funded by NIH Office of Research Infrastructure Programs (P40 OD010440).

\section{Availability of data and materials}

The datasets used and/or analyzed during the current study are available from the corresponding author on reasonable request.

Ethics approval and consent to participate

Not applicable.

\section{Consent for publication}

Not applicable.

\section{Competing interests}

None declared.

Received: 5 August 2020 Accepted: 7 October 2020

Published online: 09 November 2020

\section{References}

1. Selkoe DJ. Alzheimer's disease: genes, proteins, and therapy. Physiol Rev. 2001;81:741-66

2. Avila J, Lucas JJ, Perez M, Hernandez F. Role of tau protein in both physiological and pathological conditions. Physiol Rev. 2004;84:361-84.

3. Irwin DJ, Cohen TJ, Grossman M, Arnold SE, Xie SX, Lee VMY, Trojanowski JQ. Acetylated tau, a novel pathological signature in Alzheimer's disease and other tauopathies. Brain. 2012;135:807-18.

4. Neddens J, Temmel M, Flunkert S, Kerschbaumer B, Hoeller C, Loeffler T, Niederkofler V, Daum G, Attems J, Hutter-Paier B. Phosphorylation of different tau sites during progression of Alzheimer's disease. Acta Neuropathol Commun. 2018;6:52

5. Wu X-L, Piña-Crespo J, Zhang Y-W, Chen X-C, Xu H-X. Tau-mediated Neurodegeneration and potential implications in diagnosis and treatment of Alzheimer's disease. Chin Med J. 2017:130:2978-90.

6. Chong FP, Ng KY, Koh RY, Chye SM. Tau proteins and Tauopathies in Alzheimer's disease. Cell Mol Neurobiol. 2018:38:965-80.

7. Mi K, Johnson GWW. The role of tau phosphorylation in the pathogenesis of Alzheimer's disease. Curr Alzheimer Res. 2006:3:449-63.

8. Mroczko B, Groblewska M, Litman-Zawadzka A. The role of protein Misfolding and tau oligomers (TauOs) in Alzheimer's disease (AD). Int J Mol Sci. 2019:20:4661.

9. Augustinack JC, Schneider A, Mandelkow EM, Hyman BT. Specific tau phosphorylation sites correlate with severity of neuronal cytopathology in Alzheimer's disease. Acta Neuropathol. 2002;103:26-35.

10. Israel MA, Yuan SH, Bardy C, Reyna SM, Mu Y, Herrera C, Hefferan MP, Van Gorp S, Nazor KL, Boscolo FS, et al. Probing sporadic and familial 
Alzheimer's disease using induced pluripotent stem cells. Nature. 2012;482 216-20.

11. Schwalbe M, Kadavath H, Biernat J, Ozenne V, Blackledge M, Mandelkow E, Zweckstetter M. Structural impact of tau phosphorylation at threonine 231. Structure. 2015;23:1448-58.

12. Alonso AD, Cohen LS, Corbo C, Morozova V, Elldrissi A, Phillips G, Kleiman FE. Hyperphosphorylation of tau associates with changes in its function beyond microtubule stability. Front Cell Neurosci. 2018;12:338.

13. Sillen A, Barbier P, Landrieu I, Lefebvre S, Wieruszeski J-M, Leroy A, Peyrot V, Lippens G. NMR investigation of the interaction between the neuronal protein tau and the microtubules. Biochemistry. 2007;46:3055-64.

14. Sengupta A, Kabat J, Novak M, Wu Q, Grundke-lqbal I, labal K. Phosphorylation of tau at both Thr 231 and Ser 262 is required for maximal inhibition of its binding to microtubules. Arch Biochem Biophys. 1998;357: 299-309.

15. Shafiei SS, Guerrero-Munoz MJ, Castillo-Carranza DL. Tau oligomers: cytotoxicity, propagation, and mitochondrial damage. Front Aging Neurosci. 2017;9:83.

16. Alonso AD, Di Clerico J, Li B, Corbo CP, Alaniz ME, Grundke-labal I, labal K. Phosphorylation of tau at Thr212, Thr231, and Ser262 combined causes neurodegeneration. J Biol Chem. 2010;285:30851-60.

17. Tracy TE, Gan L. Acetylated tau in Alzheimer's disease: an instigator of synaptic dysfunction underlying memory loss: increased levels of acetylated tau blocks the postsynaptic signaling required for plasticity and promotes memory deficits associated with tauopathy. Bioessays. 2017;39. https://doi. org/10.1002/bies.201600224.

18. Min SW, Cho SH, Zhou Y, Schroeder S, Haroutunian V, Seeley WW, Huang EJ, Shen Y, Masliah E, Mukherjee C, et al. Acetylation of tau inhibits its degradation and contributes to tauopathy. Neuron. 2010;67:953-66.

19. Cohen TJ, Guo JL, Hurtado DE, Kwong LK, Mills IP, Trojanowski JQ, Lee VM The acetylation of tau inhibits its function and promotes pathological tau aggregation. Nat Commun. 2011;2:252.

20. Min S-W, Chen X, Tracy TE, Li Y, Zhou Y, Wang C, Shirakawa K, Minami SS, Defensor E, Mok SA, et al. Critical role of acetylation in taumediated neurodegeneration and cognitive deficits. Nat Med. 2015;21: 1154-62.

21. Sohn PD, Tracy TE, Son HI, Zhou Y, Leite RE, Miller BL, Seeley WW, Grinberg LT, Gan L. Acetylated tau destabilizes the cytoskeleton in the axon initial segment and is mislocalized to the somatodendritic compartment. Mol Neurodegener. 2016;11:47.

22. Ajit D, Trzeciakiewicz H, Tseng JH, Wander CM, Chen Y, Ajit A, King DP, Cohen TJ. A unique tau conformation generated by an acetylation-mimic substitution modulates P301S-dependent tau pathology and hyperphosphorylation. J Biol Chem. 2019;294:16698-711.

23. Tracy TE, Sohn PD, Minami SS, Wang C, Min SW, Li Y, Zhou Y, Le D, Lo I, Ponnusamy R, et al. Acetylated tau obstructs KIBRA-mediated signaling in synaptic plasticity and promotes Tauopathy-related memory loss. Neuron. 2016;90:245-60.

24. Hu H, Tan CC, Tan L, Yu JT. A Mitocentric view of Alzheimer's disease. Mol Neurobiol. 2017;54:6046-60.

25. Swerdlow RH. Mitochondria and mitochondrial cascades in Alzheimer's disease. J Alzheimers Dis. 2018;62:1403-16.

26. Li XC, Hu Y, Wang ZH, Luo Y, Zhang Y, Liu XP, Feng Q, Wang Q, Ye K, Liu $G P$, Wang JZ. Human wild-type full-length tau accumulation disrupts mitochondrial dynamics and the functions via increasing mitofusins. Sci Rep. 2016;6:24756.

27. David DC, Hauptmann S, Scherping I, Schuessel K, Keil U, Rizzu P, Ravid R, Drose S, Brandt U, Muller WE, et al. Proteomic and functional analyses reveal a mitochondrial dysfunction in P301L tau transgenic mice. J Biol Chem. 2005;280:23802-14.

28. Safiulina D, Kaasik A. Energetic and dynamic: how mitochondria meet neuronal energy demands. PLoS Biol. 2013;11:e1001755.

29. Mattson MP, Gleichmann M, Cheng A. Mitochondria in neuroplasticity and neurological disorders. Neuron. 2008:60:748-66.

30. Kraemer BC, Zhang B, Leverenz JB, Thomas JH, Trojanowski JQ, Schellenberg GD. Neurodegeneration and defective neurotransmission in a Caenorhabditis elegans model of tauopathy. Proc Natl Acad Sci U S A. 2003; 100:9980-5.

31. Miyasaka T, Ding Z, Gengyo-Ando K, Oue M, Yamaguchi H, Mitani S, Ihara Y. Progressive neurodegeneration in C. elegans model of tauopathy. Neurobiol Dis. 2005;20:372-83.
32. Butler VJ, Salazar DA, Soriano-Castell D, Alves-Ferreira M, Dennissen FJA, Vohra M, Oses-Prieto JA, Li KH, Wang AL, Jing B, et al. Tau/MAPT diseaseassociated variant $\mathrm{A} 152 \mathrm{~T}$ alters tau function and toxicity via impaired retrograde axonal transport. Hum Mol Genet. 2019;28:1498-514.

33. Brandt R, Gergou A, Wacker I, Fath T, Hutter H. A Caenorhabditis elegans model of tau hyperphosphorylation: induction of developmental defects by transgenic overexpression of Alzheimer's disease-like modified tau. Neurobiol Aging. 2009;30:22-33.

34. Chee F, Mudher A, Newman TA, Cuttle M, Lovestone S, Shepherd D. Overexpression of tau results in defective synaptic transmission in Drosophila neuromuscular junctions. Biochem Soc Trans. 2006;34:88-90.

35. Kosmidis S, Grammenoudi S, Papanikolopoulou K, Skoulakis EMC. Differential effects of tau on the integrity and function of neurons essential for learning in Drosophila. J Neurosci. 2010;30:464-77.

36. Maeda S, Djukic B, Taneja P, Yu G-Q, Lo I, Davis A, Craft R, Guo W, Wang $X$, Kim D, et al. Expression of A152T human tau causes agedependent neuronal dysfunction and loss in transgenic mice. EMBO Rep. 2016;17:530-51.

37. Maeda S, Sato Y, Takashima A. Frontotemporal dementia with parkinsonism linked to chromosome-17 mutations enhance tau oligomer formation. Neurobiol Aging. 2018;69:26-32.

38. Miyasaka T, Shinzaki Y, Yoshimura S, Yoshina S, Kage-Nakadai E, Mitani S, Ihara Y. Imbalanced Expression of Tau and Tubulin Induces Neuronal Dysfunction in C. elegans Models of Tauopathy. Front Neurosci. 2018;12:415.

39. Fang EF, Hou Y, Palikaras K, Adriaanse BA, Kerr JS, Yang B, Lautrup S, HasanOlive MM, Caponio D, Dan $X$, et al. Mitophagy inhibits amyloid- $\beta$ and tau pathology and reverses cognitive deficits in models of Alzheimer's disease. Nat Neurosci. 2019;22:401-12.

40. Wang C, Saar V, Leung KL, Chen L, Wong G. Human amyloid $\beta$ peptide and tau co-expression impairs behavior and causes specific gene expression changes in Caenorhabditis elegans. Neurobiol Dis. 2018;109:88-101.

41. Frokjaer-Jensen C, Davis MW, Sarov M, Taylor J, Flibotte S, LaBella M, Pozniakovsky A, Moerman DG, Jorgensen EM. Random and targeted transgene insertion in Caenorhabditis elegans using a modified Mos1 transposon. Nat Methods. 2014;11:529-34.

42. Chalfie M, Thomson JN. Structural and functional diversity in the neuronal microtubules of Caenorhabditis elegans. J Cell Biol. 1982;93:15-23.

43. Prasanth MI, Gayathri S, Bhaskar JP, Krishnan V, Balamurugan K. Analyzing the Synergistic Effects of Antioxidants in Combating Photoaging Using Model Nematode, Caenorhabditis elegans. Photochem Photobiol. 2019. https://doi.org/10.1111/php.13167.

44. Sagi D, Kim SK. An engineering approach to extending lifespan in C. elegans. PLoS Genet. 2012;8:e1002780.

45. Gurskaya NG, Verkhusha W, Shcheglov AS, Staroverov DB, Chepurnykh TV, Fradkov AF, Lukyanov S, Lukyanov KA. Engineering of a monomeric greento-red photoactivatable fluorescent protein induced by blue light. Nat Biotechnol. 2006;24:461-5.

46. Nehrke K, Begenisich T, Pilato J, Melvin JE. Into ion channel and transporter function. Caenorhabditis elegans ClC-type chloride channels: novel variants and functional expression. Am J Phys Cell Phys. 2000;279:C2052-66.

47. Frokjaer-Jensen C, Davis MW, Hopkins CE, Newman BJ, Thummel JM, Olesen SP, Grunnet M, Jorgensen EM. Single-copy insertion of transgenes in Caenorhabditis elegans. Nat Genet. 2008;40:1375-83.

48. Kim H, Ishidate T, Ghanta KS, Seth M, Conte D Jr, Shirayama M, Mello CC. A Co-CRISPR strategy for efficient genome editing in Caenorhabditis elegans. Genetics. 2014;197:1069-80.

49. Paix A, Folkmann A, Rasoloson D, Seydoux G. High efficiency, homologydirected genome editing in Caenorhabditis elegans using CRISPR-Cas9 Ribonucleoprotein complexes. Genetics. 2015;201:47-54.

50. Brenner S. The genetics of Caenorhabditis elegans. Genetics. 1974;77:71-94.

51. Kim H, Calatayud C, Guha S, Fernandez-Carasa I, Berkowitz L, CarballoCarbajal I, Ezquerra M, Fernandez-Santiago R, Kapahi P, Raya A, et al. The small GTPase RAC1/CED-10 is essential in maintaining dopaminergic neuron function and survival against alpha-Synuclein-induced toxicity. Mol Neurobiol. 2018;55:7533-52.

52. Wang J, Farr GW, Hall DH, Li F, Furtak K, Dreier L, Horwich AL. An ALS-linked mutant SOD1 produces a locomotor defect associated with aggregation and synaptic dysfunction when expressed in neurons of Caenorhabditis elegans. PLoS Genet. 2009;5:e1000350.

53. Chen $X$, Chalfie M. Modulation of $C$. elegans Touch Sensitivity Is Integrated at Multiple Levels. J Neurosci. 2014;34:6522-36. 
54. Chatzigeorgiou M, Grundy L, Kindt KS, Lee W-H, Driscoll M, Schafer WR. Spatial asymmetry in the mechanosensory phenotypes of the $C$. elegans DEG/ENaC gene mec-10. J Neurophysiol. 2010;104:3334-44.

55. Sutphin GL, Kaeberlein M. Measuring Caenorhabditis elegans life span on solid media. J Vis Exp. 2009. https://doi.org/10.3791/1152.

56. Charmpilas N, Kounakis K, Tavernarakis N. Monitoring Mitophagy during aging in Caenorhabditis elegans. Methods Mol Biol. 1759;2018:151-60.

57. Palikaras K, Tavernarakis N. Assessing mitochondrial selective autophagy in the nematode Caenorhabditis elegans. Methods Mol Biol. 2017;1567:349-61.

58. Chen $\mathrm{C}-\mathrm{H}$, Chen $\mathrm{Y}-\mathrm{C}$, Jiang $\mathrm{H}-\mathrm{C}$, Chen $\mathrm{C}-\mathrm{K}$, Pan C-L. Neuronal aging: learning from C. elegans. J Mol Signal. 2013;8:14.

59. Gallegos ME, Bargmann Cl. Mechanosensory Neurite termination and tiling depend on SAX-2 and the SAX-1 kinase. Neuron. 2004;44:239-49.

60. Marcette JD, Chen JJ, Nonet ML. The Caenorhabditis elegans microtubule minus-end binding homolog PTRN-1 stabilizes synapses and neurites. Elife. 2014;3:e01637

61. Sun N, Malide D, Liu J, Rovira II, Combs CA, Finkel T. A fluorescence-based imaging method to measure in vitro and in vivo mitophagy using mtKeima. Nat Protoc. 2017;12:1576-87.

62. Williams JA, Zhao K, Jin S, Ding W-X. New methods for monitoring mitochondrial biogenesis and mitophagy in vitro and in vivo. Exp Biol Med. 2017;242:781-7.

63. Jiang $\mathrm{H}-\mathrm{C}$, Hsu J-M, Yen C-P, Chao C-C, Chen R-H, Pan C-L. Neural activity and CaMKII protect mitochondria from fragmentation in agingCaenorhabditis elegansneurons. Proc Natl Acad Sci. 2015;112:8768-73.

64. Kolarova M, Garcia-Sierra F, Bartos A, Ricny J, Ripova D. Structure and pathology of tau protein in Alzheimer disease. Int J Alzheimers Dis. 2012; 2012:731526.

65. Dregni AJ, Mandala VS, Wu H, Elkins MR, Wang HK, Hung I, DeGrado WF, Hong M. In vitro 0N4R tau fibrils contain a monomorphic $\beta$-sheet core enclosed by dynamically heterogeneous fuzzy coat segments. Proc Natl Acad Sci U S A. 2019:116:16357-66.

66. Manczak M, Calkins MJ, Reddy PH. Impaired mitochondrial dynamics and abnormal interaction of amyloid beta with mitochondrial protein Drp1 in neurons from patients with Alzheimer's disease: implications for neuronal damage. Hum Mol Genet. 2011;20:2495-509.

67. Reddy PH, Manczak M, Yin X. Mitochondria-division inhibitor 1 protects against amyloid- $\beta$ induced mitochondrial fragmentation and synaptic damage in Alzheimer's disease. J Alzheimers Dis. 2017;58:147-62.

68. Kandimalla R, Manczak M, Fry D, Suneetha Y, Sesaki H, Reddy PH. Reduced dynamin-related protein 1 protects against phosphorylated tau-induced mitochondrial dysfunction and synaptic damage in Alzheimer's disease. Hum Mol Genet. 2016:25:4881-97.

69. Cai Q, Tammineni P. Alterations in mitochondrial quality control in Alzheimer's disease. Front Cell Neurosci. 2016;10:24

70. Hamacher-Brady A, Brady NR. Mitophagy programs: mechanisms and physiological implications of mitochondrial targeting by autophagy. Cell Mol Life Sci. 2016;73:775-95.

71. Martinez-Vicente M. Neuronal Mitophagy in neurodegenerative diseases. Front Mol Neurosci. 2017;10:64.

72. Wang Y, Liu N, Lu B. Mechanisms and roles of mitophagy in neurodegenerative diseases. CNS Neurosci Ther. 2019;25:859-75.

73. Chang JT, Kumsta C, Hellman AB, Adams LM, Hansen M. Spatiotemporal regulation of autophagy during Caenorhabditis elegans aging. Elife. 2017;6: e18459.

74. Manil-Ségalen M, Lefebvre $C$, Jenzer $C$, Trichet $M$, Boulogne C, SatiatJeunemaitre $B$, Legouis $R$. The $C$. elegans LC3 acts downstream of GABARAP to degrade autophagosomes by interacting with the HOPS subunit VPS39. Dev Cell. 2014:28:43-55.

75. Cenini G, Voos W. Mitochondria as potential targets in Alzheimer disease therapy: an update. Front Pharmacol. 2019;10:902.

76. Santacruz K, Lewis J, Spires T, Paulson J, Kotilinek L, Ingelsson M, Guimaraes A, DeTure M, Ramsden M, McGowan E, et al. Tau suppression in a neurodegenerative mouse model improves memory function. Science. 2005;309:476-81.

77. Davis M, T OC, Johnson S, Cline S, Merikle E, Martenyi F, Simpson K. Estimating Alzheimer's disease progression rates from Normal cognition through mild cognitive impairment and stages of dementia. Curr Alzheimer Res. 2018;15:777-88.

78. Fatouros C, Pir GJ, Biernat J, Koushika SP, Mandelkow E, Mandelkow EM, Schmidt E, Baumeister R. Inhibition of tau aggregation in a novel
Caenorhabditis elegans model of tauopathy mitigates proteotoxicity. Hum Mol Genet. 2012;21:3587-603.

79. Cho JH, Johnson GV. Primed phosphorylation of tau at Thr231 by glycogen synthase kinase 3beta (GSK3beta) plays a critical role in regulating tau's ability to bind and stabilize microtubules. J Neurochem. 2004;88:349-58.

80. Xia D, Li C, Götz J. Pseudophosphorylation of tau at distinct epitopes or the presence of the P301L mutation targets the microtubule-associated protein tau to dendritic spines. Biochim Biophys Acta. 1852;2015:913-24.

81. Ułamek-Kozioł M, Furmaga-Jabłońska W, Januszewski S, Brzozowska J, Sciślewska M, Jabłoński M, Pluta R. Neuronal autophagy: self-eating or selfcannibalism in Alzheimer's disease. Neurochem Res. 2013;38:1769-73.

82. Mondragón-Rodríguez S, Perry G, Zhu X, Moreira Pl, Acevedo-Aquino MC, Williams S. Phosphorylation of tau protein as the link between oxidative stress, mitochondrial dysfunction, and connectivity failure: implications for Alzheimer's disease. Oxidative Med Cell Longev. 2013;2013:940603.

83. Quintanilla RA, Dolan PJ, Jin YN, Johnson GV. Truncated tau and Abeta cooperatively impair mitochondria in primary neurons. Neurobiol Aging. 2012;33:619.e625-35.

84. Kerr JS, Adriaanse BA, Greig NH, Mattson MP, Cader MZ, Bohr VA, Fang EF. Mitophagy and Alzheimer's disease: cellular and molecular mechanisms. Trends Neurosci. 2017:40:151-66.

85. Kraemer BC, Burgess JK, Chen JH, Thomas JH, Schellenberg GD. Molecular pathways that influence human tau-induced pathology in Caenorhabditis elegans. Hum Mol Genet. 2006;15:1483-96.

86. Cornelissen T, Vilain S, Vints K, Gounko N, Verstreken P, Vandenberghe W. Deficiency of parkin and PINK1 impairs age-dependent mitophagy in Drosophila. eLife. 2018;7:e35878.

87. Yeo D, Kang C, Gomez-Cabrera MC, Vina J, Ji LL. Intensified mitophagy in skeletal muscle with aging is downregulated by PGC-1alpha overexpression in vivo. Free Radic Biol Med. 2019;130:361-8.

88. Hou X, Fiesel FC, Truban D, Castanedes Casey M, Lin W-L, Soto Al, Tacik P, Rousseau LG, Diehl NN, Heckman MG, et al. Age- and disease-dependent increase of the mitophagy marker phospho-ubiquitin in normal aging and Lewy body disease. Autophagy. 2018;14:1404-18.

89. Drake JC, Yan Z. Mitophagy in maintaining skeletal muscle mitochondrial proteostasis and metabolic health with ageing. J Physiol. 2017;595:6391-9.

90. Fivenson EM, Lautrup S, Sun N, Scheibye-Knudsen M, Stevnsner T, Nilsen H, Bohr VA, Fang EF. Mitophagy in neurodegeneration and aging. Neurochem Int. 2017;109:202-9.

91. Chakravorty A, Jetto CT, Manjithaya R. Dysfunctional mitochondria and Mitophagy as drivers of Alzheimer's disease pathogenesis. Front Aging Neurosci. 2019:11:311.

92. Reddy PH, Oliver DM. Amyloid Beta and Phosphorylated tau-induced defective autophagy and Mitophagy in Alzheimer's disease. Cells. 2019;8:488.

93. Fang EF, Hou Y, Lautrup S, Jensen MB, Yang B, SenGupta T, Caponio D, Khezri R, Demarest TG, Aman $Y$, et al. NAD(+) augmentation restores mitophagy and limits accelerated aging in Werner syndrome. Nat Commun. 2019:10:5284.

94. Flannery PJ, Trushina E. Mitochondrial dynamics and transport in Alzheimer's disease. Mol Cell Neurosci. 2019:98:109-20.

95. Cummins N, Tweedie A, Zuryn S, Bertran-Gonzalez J, Götz J. Diseaseassociated tau impairs mitophagy by inhibiting Parkin translocation to mitochondria. EMBO J. 2019:38:e99360.

96. Palikaras K, Lionaki E, Tavernarakis N. Coordination of mitophagy and mitochondrial biogenesis during ageing in C. elegans. Nature. 2015;521: $525-8$.

97. Melber A, Haynes CM. UPR(mt) regulation and output: a stress response mediated by mitochondrial-nuclear communication. Cell Res. 2018;28: 281-95.

98. Cooper JF, Machiela E, Dues DJ, Spielbauer KK, Senchuk MM, Van Raamsdonk JM. Activation of the mitochondrial unfolded protein response promotes longevity and dopamine neuron survival in Parkinson's disease models. Sci Rep. 2017;7:16441.

99. Martinez BA, Petersen DA, Gaeta AL, Stanley SP, Caldwell GA, Caldwell KA. Dysregulation of the Mitochondrial Unfolded Protein Response Induces Non-Apoptotic Dopaminergic Neurodegeneration in C. elegans Models of Parkinson's Disease. J Neurosci. 2017;37:11085-100.

\section{Publisher's Note}

Springer Nature remains neutral with regard to jurisdictional claims in published maps and institutional affiliations. 